Алгебра и анализ

Том. 17 (2005), вып. 4
St. Petersburg Math. J. Vol. 17 (2006), No. 4, Pages 553-579 S 1061-0022(06)00920-4

Article electronically published on May 3, 2006

\title{
ON ZETA FUNCTIONS OF ORTHOGONAL GROUPS OF SINGLE-CLASS POSITIVE DEFINITE QUADRATIC FORMS
}

\author{
A. ANDRIANOV
}

\begin{abstract}
Representations of Hecke-Shimura rings of integral single-class positive definite quadratic forms on relevant spaces of harmonic forms are considered, and the problem of simultaneous diagonalization of the corresponding Hecke operators is investigated. Explicit relations are deduced between zeta functions of the single-class quadratic forms in two and four variables corresponding to the harmonic eigenforms of genus 1 and 2, respectively, and zeta functions of the theta-series weighted by these eigenforms.
\end{abstract}

\section{INTRODUCTION}

By definition an (integral proper) automorph of a nonsingular integral quadratic form $\mathbf{q}(X)$ in $m$ variables is an integral $(m \times m)$-matrix $D$ having positive determinant and satisfying the condition

$$
\mathbf{q}(D X)=\mu \mathbf{q}(X) \quad\left({ }^{t} X=\left(x_{1}, \ldots, x_{m}\right)\right) ;
$$

the number $\mu=\mu(D)$ is the multiplier of the automorph. All automorphs form a semigroup $\mathbf{A}=A(\mathbf{q})$, the automorph semigroup of $\mathbf{q}$, and the automorphs with multiplier $\mu=1$ form a subgroup $\mathbf{E}=E(\mathbf{q})$, called the group of (proper) units of $\mathbf{q}$. The HeckeShimura ring or the automorph class ring of $\mathbf{q}$ over $\mathbb{Z}$ consists of all finite formal linear combinations with coefficients in $\mathbb{Z}$ of the symbols $(\mathbf{E} D \mathbf{E})=\tau(D)$ corresponding in a one-to-one way to the double cosets $\mathbf{E} D \mathbf{E}$ of $\mathbf{A}$ modulo $\mathbf{E}$, also called the double cosets,

$$
\mathcal{H}=\mathcal{H}(\mathbf{q})=\left\{\tau=\sum_{\alpha} a_{\alpha} \tau\left(D_{\alpha}\right) \mid a_{\alpha} \in \mathbb{Z}, D_{\alpha} \in \mathbf{A}\right\},
$$

where the product of two double cosets is defined by

$$
\tau(D) \tau\left(D^{\prime}\right)=\sum_{\mathbf{E} D^{\prime \prime} \mathbf{E} \subset \mathbf{E} D \mathbf{E} D^{\prime} \mathbf{E}} c\left(D, D^{\prime} ; D^{\prime \prime}\right) \tau\left(D^{\prime \prime}\right),
$$

and $c\left(D, D^{\prime} ; D^{\prime \prime}\right)$ is the number of pairs of representatives $D_{i} \in \mathbf{E} \backslash \mathbf{E} D \mathbf{E}$ and $D_{j}^{\prime} \in$ $\mathbf{E} \backslash \mathbf{E} D^{\prime} \mathbf{E}$ satisfying $D_{i} D_{j}^{\prime} \in \mathbf{E} D^{\prime \prime}$. Generally speaking, little can be said about the ring $\mathcal{H}$, and it should be replaced by a more complicated construction of a matrix HeckeShimura ring, which we do not touch upon in this paper. However, in some cases, for example, if the form $\mathbf{q}$ belongs to a single-class genus, the ring $\mathcal{H}$ has many nice properties

2000 Mathematics Subject Classification. Primary 11F27, 11F46, 11F60, 14G10, $20 \mathrm{C} 08$.

Key words and phrases. Harmonic forms, Hecke-Shimura rings, Hecke operators, modular forms in one and several variables, theta-series of integral quadratic forms, zeta functions of quadratic forms, zeta functions of modular forms.

Supported in part by the RFBR (grant no. 05-01-00930). 
and can be considered in its own right. In particular, the sums of double cosets of fixed multipliers,

$$
\tau(\mu)=\sum_{D \in \mathbf{E} \backslash \mathbf{A} / \mathbf{E}, \mu(D)=\mu} \tau(D) \in \mathcal{H},
$$

satisfy the relations

$$
\tau(\mu) \tau(\nu)=\tau(\nu) \tau(\mu)=\tau(\mu \nu)
$$

if $\mu$ and $\nu$ are coprime and $\mu$ or $\nu$ is coprime to the determinant of $\mathbf{q}$. It follows that the formal Dirichlet series with the coefficients $\tau(1), \tau(2), \ldots$ (note that $\tau(1)=\tau\left(1_{m}\right)$ is the unity element of the ring $\mathcal{H}$ ) can be expanded in a formal Euler product:

$$
\sum_{\mu=1}^{\infty} \frac{\tau(\mu)}{\mu^{s}}=\sum_{\nu \mid(\operatorname{det} \mathbf{q})^{\infty}} \frac{\tau(\nu)}{\nu^{s}} \prod_{p \nmid \operatorname{det} \mathbf{q}} \sum_{\delta=0}^{\infty} \tau\left(p^{\delta}\right) p^{-\delta s},
$$

where $\mu^{s}$ is viewed merely as a formal quasicharacter of the multiplicative semigroup $\mathbb{N}$ of positive integers, and $\nu$ and $p$ range (respectively) over all positive integers dividing some powers of det $\mathbf{q}$ and all prime numbers not dividing det $\mathbf{q}$. In a more general situation, in An(94) it was conjectured that, for each prime number $p$ not dividing det $\mathbf{q}$, the formal power series with coefficients $\tau(1), \tau(p), \tau\left(p^{2}\right), \ldots$ is (formally) a rational fraction over the ring $\mathcal{H}=\mathcal{H}(\mathbf{q})$ with denominator of degree $2^{k}$ and with numerator of degree at most $2^{k}-2$, provided the number $m$ of the variables of $\mathbf{q}$ equals $2 k-1$ or $2 k$ :

$$
\sum_{\delta=0}^{\infty} \tau\left(p^{\delta}\right) t^{\delta}=R_{p}(t)^{-1} S_{p}(t)
$$

where

$$
R_{p}(t)=\tau(1)+\sum_{1 \leq i \leq 2^{k}} \rho_{i} t^{i}, \quad S_{p}(t)=\sum_{0 \leq j \leq 2^{k}-2} \sigma_{j} t^{j}
$$

with $\rho_{i}=\rho_{i}(p), \sigma_{j}=\sigma_{j}(p) \in \mathcal{H}(\mathbf{q})$. In this case we shall say that the formal power series over $\mathcal{H}(\mathbf{q})$ given by

$$
Z_{p}(t, \mathbf{q})=R_{p}(t)^{-1}
$$

is a local zeta-series of q. In $\mathrm{An}(94)$ it was proved that this conjecture is true for the quadratic forms in $m=2,3$, and 4 variables.

Now, suppose we are given a complex representation $\tau \mapsto \mid \tau$ of the $\operatorname{ring} \mathcal{H}(\mathbf{q})$, and $P$ is a common eigenvector, so that $P \mid \tau=\lambda(\tau) P$ with eigenvalues $\lambda(\tau)$. Then, by analogy with the theory of zeta functions of Siegel modular forms, we can consider the power series

$$
Z_{p}(t, P)=\left(1+\sum_{1 \leq i \leq 2^{k}} \lambda\left(\rho_{i}\right) t^{i}\right)^{-1}
$$

and the Euler product

$$
Z^{*}(s, P)=\prod_{p \nmid \operatorname{det} \mathbf{q}} Z_{p}\left(p^{-s}, P\right),
$$

which can naturally be called (respectively) the local and the (regular) global orthogonal zeta function of the form $\mathbf{q}$ corresponding to the eigenvector $P$, and we can ask about the properties of these zeta functions. Below we shall show that in some cases the orthogonal zeta functions can be expressed explicitly in terms of spinor zeta functions of appropriate Siegel modular forms.

In $\S 1$ we present a survey of the basic definitions and properties of the automorph class rings. In $\S 2$ we examine the standard representations of automorph class rings on spaces of harmonic forms and study the existence of eigenfunctions. In $\S 3$, in particular, we find 
explicit expressions of the zeta functions of single-class positive definite quadratic forms in $m=2$ and 4 variables corresponding to these eigenfunctions in terms of the Hecke zeta function if $m=2$ and, if $m=4$, in terms of the Andrianov zeta function of genus 2 of harmonic theta-series with the harmonic eigenfunctions as coefficients (Theorems 3.3 and 3.4, respectively). By examples of binary quadratic forms of discriminants -4 and -3 , in $\S 4$ it is shown that the corresponding global zeta functions coincide, in fact, with the zeta functions of relevant quadratic fields with appropriate Hecke characters.

The assumption that the quadratic forms under consideration are single-class can be lifted. My next paper will be devoted to this problem.

There is little doubt that the results of rather elaborate calculations presented here reflect much more general links between automorphic representations of Hecke-Shimura algebras of orthogonal and symplectic groups over global fields. The transformation formalism that expresses the images of harmonic theta-series under the action of Hecke operators in terms of the action of automorph class rings on their harmonic coefficients, as well as the underlying relations between the Hecke-Shimura rings of symplectic groups and the automorph class rings, may provide an initial tool for investigation of these links (see, e.g., $\operatorname{An}(91)],[\operatorname{An}(92)]$, and $\operatorname{An}(96)]$. Nevertheless, direct approaches to orthogonal zeta functions, not based on reduction to the symplectic case, would also be of considerable interest.

Notation. As usual, we reserve the letters $\mathbb{N}, \mathbb{Z}, \mathbb{Q}$, and $\mathbb{C}$ for the set of positive rational integers, the ring of rational integers, the field of rational numbers, and the field of complex numbers, respectively.

If $\mathbb{A}$ is a set, $\mathbb{A}_{n}^{m}$ denotes the set of all $(m \times n)$-matrices with elements in $\mathbb{A}$. If $\mathbb{A}$ is a ring with identity element, then $1_{n}$ denotes the identity element of the ring $\mathbb{A}_{n}^{n}$. The transpose of a matrix $M$ is denoted by ${ }^{t} M$. For two matrices $S$ and $N$ of appropriate sizes, we write

$$
S[N]={ }^{t} N S N .
$$

\section{Automorph ClASS RINGS OF SINGLE-CLASS QUADRATIC FORMS}

A quadratic form

$$
\mathbf{q}(X)=\frac{1}{2} Q[X] \quad\left({ }^{t} X=\left(x_{1}, \ldots, x_{m}\right)\right)
$$

in $m$ variables with matrix $Q$ is said to be integral if its matrix $Q$ belongs to the set

$$
\mathbb{E}_{m}=\left\{Q=\left(Q_{i j}\right) \in \mathbb{Z}_{m}^{m} \mid Q_{i i} \in 2 \mathbb{Z} ; Q_{i j}=Q_{j i}(i, j=1, \ldots, m)\right\}
$$

of even matrices of order $m$. Instead of the language of integral quadratic forms, we shall mainly use the equivalent language of even matrices. We recall that two even matrices $Q$ and $Q^{\prime}$ of order $m$ are said to be (properly) equivalent, $Q \cong Q^{\prime}$, if

$$
Q^{\prime}=Q[U] \quad \text { with some } U \in \Lambda=\Lambda^{m}=S L_{m}(\mathbb{Z}) .
$$

The set

$$
\{Q\}=\left\{Q^{\prime}=Q[U] \mid U \in \Lambda^{m}\right\}
$$

of all matrices $Q^{\prime}$ equivalent to a given matrix $Q$ is called the (proper) equivalence class of $Q$. The basic characteristics of an integral quadratic form $\mathbf{q}$ with matrix $Q$, such as the signature (i.e., the number of positive and negative squares in a real diagonalization of $\mathbf{q}$ ), the determinant $d=d(\mathbf{q})=\operatorname{det} Q$, the divisor (for $\mathbf{q} \neq 0$; this is the largest natural number $\delta$ such that $\delta^{-1} Q$ is an even matrix), and the level (for $\operatorname{det} Q \neq 0$; this is the smallest natural number $q$ such that $q Q^{-1}$ is an even matrix), all depend only on the equivalence class of the matrix $Q$. In accordance with the reduction theory of 
integral quadratic forms (see, e.g., $\mathrm{Ca}(78)$, Chapter 9]), the set of all even matrices of fixed size and fixed nonzero determinant is the union of finitely many classes of integrally equivalent matrices. We say that an even nonsingular matrix $Q$ is a single-class matrix if all the matrices having the same signature, divisor, level, and determinant as those of $Q$ belong to the equivalence class $\{Q\}$.

We fix a nonsingular matrix $Q \in \mathbb{E}_{m}$. Then condition (0.1) means that $Q[D]=\mu Q$, and we denote by

$$
\mathbf{A}(\mu)=A(Q, \mu)=\left\{D \in \mathbb{Z}_{m}^{m} \mid \operatorname{det} D>0, Q[D]=\mu Q\right\}
$$

the set of (integral proper) automorphs of the matrix $Q$ with multiplier $\mu$. The set

$$
\mathbf{E}=E(Q)=A(Q, 1)
$$

is called the group of (proper) units of $Q$. It is clear that

$$
\mathbf{A}(\mu) \mathbf{A}(\nu) \subset \mathbf{A}(\mu \nu) \text { and } \quad \mathbf{E A}(\mu) \mathbf{E}=\mathbf{A}(\mu) .
$$

The set

$$
\mathbf{A}=A(Q)=\bigcup_{\mu=1}^{\infty} A(Q, \mu)
$$

is the semigroup of automorphs of $Q$, and

$$
\mathbf{A}_{\text {reg }}=A_{\text {reg }}(Q)=\bigcup_{\mu \geq 1, \operatorname{gcd}(\mu, \operatorname{det} Q)=1} A(Q, \mu)
$$

denotes the semigroup of regular automorphs of $Q$.

Lemma 1.1. The mapping

$$
D \mapsto D^{*}=\mu(D) D^{-1} \quad\left(D \in A_{\text {reg }}(Q)\right)
$$

is one-to-one on each subset $A(Q, \mu) \subset A_{\mathrm{reg}}(Q)$ and determines a second-order antiautomorphism of the semigroup $A_{\mathrm{reg}}(Q)$.

Proof. If $D \in A(Q, \mu) \subset A_{\text {reg }}(Q)$, then $Q[D]={ }^{t} D Q D=\mu Q$ and $\operatorname{gcd}(\mu, \operatorname{det} Q)=1$. It follows that $D^{*}=\mu D^{-1}=Q^{-1}{ }^{t} D Q$, and hence the matrix $D^{*}$ is integral, because its products by coprime numbers $\operatorname{det} D=\mu^{m / 2}$ and $\operatorname{det} Q$ are integral matrices. Next, clearly, we have $Q\left[D^{*}\right]=\mu Q$, whence $D^{*} \in A(Q, \mu)$ and $D^{* *}=D$. Finally, by (1.1),

$$
\left(D D_{1}\right)^{*}=\mu\left(D D_{1}\right)\left(D D_{1}\right)^{-1}=\mu\left(D_{1}\right)\left(D_{1}\right)^{-1} \mu(D) D^{-1}=D_{1}^{*} D^{*} \text {. }
$$

Next, we introduce the automorph class ring $\mathcal{H}=\mathcal{H}(Q)$ of $Q($ over $\mathbb{C})$ as the automorph class ring (0.2) of the quadratic form with matrix $Q$, and denote by

$$
\mathcal{H}_{\text {reg }}=\mathcal{H}_{\text {reg }}(Q) \subset \mathcal{H}(Q)
$$

the regular automorph class ring of $Q$ consisting of all finite linear combinations of the double cosets of regular automorphs.

Lemma 1.2. For each nonsingular matrix $D \in \mathbb{Z}_{m}^{m}$, the intersection $\Lambda^{m} D \cap A(Q)$ either is empty, or else consists of a unique left coset $E(Q) D^{\prime}$ of the semigroup $A(Q)$ relative to the group $E(Q)$. In particular, each double coset $E(Q) D E(Q)$ contained in $A(Q)$ is a finite union of left cosets modulo $E(Q)$.

Proof. Indeed, if $D^{\prime}, D^{\prime \prime} \in \mathbf{A} \cap \Lambda D$, then $D^{\prime \prime}=U D^{\prime}$ with $U \in \Lambda$ and

$$
Q[U]=Q\left[D^{\prime \prime}\left(D^{\prime}\right)^{-1}\right]=\mu^{\prime \prime}\left(\mu^{\prime}\right)^{-1} Q=Q,
$$

where $\mu^{\prime \prime}=\mu\left(D^{\prime \prime}\right)=\mu\left(D^{\prime}\right)=\mu^{\prime}$, whence $U \in \mathbf{E}$ and $D^{\prime \prime} \in \mathbf{E} D^{\prime}$. The second assertion follows, since it is well known that the number of left cosets $\Lambda \backslash \Lambda D \Lambda$ is finite for every integral nonsingular $D$. 
It will be convenient to write each double $\operatorname{coset} \tau(D) \in \mathcal{H}$ as a formal linear combination of different left cosets contained in $\mathbf{E} D \mathbf{E}$,

$$
\tau(D)=\sum_{D_{i} \in \mathbf{E} \backslash \mathbf{E} D \mathbf{E}}\left(\mathbf{E} D_{i}\right) \quad(D \in \mathbf{A}) .
$$

Then the multiplication of double cosets defined in the Introduction can be written in the form

$$
\tau(D) \tau\left(D^{\prime}\right)=\sum_{D_{i} \in \mathbf{E} \backslash \mathbf{E} D \mathbf{E}}\left(\mathbf{E} D_{i}\right) \sum_{D_{j}^{\prime} \in \mathbf{E} \backslash \mathbf{E} D^{\prime} \mathbf{E}}\left(\mathbf{E} D_{j}^{\prime}\right)=\sum_{D_{i}, D_{j}^{\prime}}\left(\mathbf{E} D_{i} D_{j}^{\prime}\right) .
$$

The following proposition extends the antiautomorphism defined in Lemma 1.1 to regular automorph class rings.

Proposition 1.3. The map defined on the linear combinations of double cosets by the rule

$$
\mathcal{H}_{\mathrm{reg}}(Q) \ni \tau=\sum_{i} a_{i} \tau\left(D_{i}\right) \mapsto \tau^{*}=\sum_{i} a_{i} \tau\left(D_{i}^{*}\right),
$$

where the star stands for the mapping (1.5), is a linear second-order antiautomorphism of the regular ring $\mathcal{H}_{\mathrm{reg}}(Q)$.

Proof. By Lemma 1.1 and the definition, the mapping (1.8) is a linear mapping of the ring $\mathcal{H}_{\text {reg }}$ into itself, and $\left(\tau^{*}\right)^{*}=\tau$. In particular, it is one-to-one.

It remains to prove that the map is a multiplicative antihomomorphism, i.e., it satisfies

$$
\left(\tau \tau_{1}\right)^{*}=\tau_{1}^{*} \tau^{*} \quad\left(\tau, \tau_{1} \in \mathcal{H}_{\text {reg }}\right) .
$$

We extend the ring $\mathcal{H}_{\text {reg }}$ to a similar ring $\mathcal{H}^{\prime}$ consisting of all formal finite linear combinations with integral coefficients of double cosets modulo the group $\mathbf{E}=E(Q)$ of all matrices $D \in \mathbb{Q}_{m}^{m}$ satisfying the relations $Q[D]=\mu(D) Q$ with $\mu(Q)>0$, where multiplication is defined again by (1.7). By $\operatorname{An}(87)$, Proposition 3.1.7], the linear mapping of the ring $\mathcal{H}^{\prime}$ into itself defined on the double classes $\mathbf{E} D \mathbf{E}$ by $\mathbf{E} D \mathbf{E} \mapsto \mathbf{E} D^{-1} \mathbf{E}$ is an antiautomorphism of the ring. Moreover, clearly, the linear mapping of the ring $\mathcal{H}^{\prime}$ into itself defined on the double classes by $\mathbf{E} D \mathbf{E} \mapsto \mathbf{E} \mu\left(D^{-1}\right) D \mathbf{E}$ is a homomorphic embedding. It follows that the mapping $\tau \mapsto \tau^{*}$, which can be regarded as the restriction to the subring $\mathcal{H}_{\text {reg }} \subset \mathcal{H}^{\prime}$ of the composition of these two mappings, satisfies the required condition.

In accordance with the theory of elementary divisors for the group $\Lambda=\Lambda^{m}$ (see An(87), §3.2.1] for the case of the group $G L_{m}(\mathbb{Z})$; the case of the special linear group is quite similar), for each matrix $D \in \mathbb{Z}_{m}^{m}$ with positive determinant $d=\operatorname{det} D$ there exist unique positive integers $d_{1}=d_{1}(D), \ldots, d_{m}=d_{m}(D)$, the elementary divisors of $D$, that satisfy the conditions

$$
d_{1}\left|d_{2}\right| \cdots \mid d_{m} \quad \text { and } \quad D \in \Lambda \operatorname{diag}\left(d_{1}, \ldots, d_{m}\right) \Lambda .
$$

The elementary divisors of $D$ depend only on the double coset $\Lambda D \Lambda$, and

$$
d_{i}(D) d_{i}\left(D^{\prime}\right)=d_{i}\left(D D^{\prime}\right) \quad(i=1, \ldots, m) \quad \text { if } \operatorname{gcd}\left(\operatorname{det} D, \operatorname{det} D^{\prime}\right)=1 .
$$

We denote by

$$
\mathcal{D}(D)=\operatorname{diag}\left(d_{1}(D), \ldots, d_{m}(D)\right)
$$

the diagonal matrix of elementary divisors of $D$. 
Lemma 1.4. The elementary divisors of an automorph $D \in A_{\mathrm{reg}}(Q)$ of a nonsingular even matrix $Q$ satisfy the relations

$$
\mathcal{D}(D)=\mathcal{D}\left(\mu(D) D^{-1}\right) \Longleftrightarrow d_{i}(D) d_{m-i+1}(D)=\mu(D) \quad(i=1, \ldots, m) .
$$

Proof. Since $\operatorname{det} D=\mu(D)^{m / 2}$ is coprime to $\operatorname{det} Q$, writing the relation ${ }^{t} D Q D=\mu(D) Q$ in the form ${ }^{t} D Q=Q \cdot \mu(D) D^{-1}$ and using $(1.10)$, we see that $\mathcal{D}\left({ }^{t} D\right) \mathcal{D}(Q)=\mathcal{D}(D) \mathcal{D}(Q)=$ $\mathcal{D}(Q) \mathcal{D}\left(\mu(D) D^{-1}\right)$, which implies (1.12).

For positive integers $d_{1}, \ldots, d_{m}$ satisfying $d_{1}\left|d_{2}\right| \cdots \mid d_{m}$ and $d_{i} d_{m-i+1}=\mu$ for $i=$ $1, \ldots, m$, we introduce the following elements of the ring $\mathcal{H}=\mathcal{H}(Q)$ :

$$
\tau\left[d_{1}, \ldots, d_{m}\right]=\sum_{\substack{D \in \mathbf{E} \backslash A(Q) / \mathbf{E} ; \\ \mathcal{D}(D)=\operatorname{diag}\left(d_{1}, \ldots, d_{m}\right)}} \tau(D)
$$

and

$$
\begin{aligned}
\tau(\mu) & =\sum_{\substack{d_{1}|\cdots| d_{m} ; \\
d_{i} d_{m-i+1}=\mu}} \tau\left[d_{1}, \ldots, d_{m}\right] \\
& =\sum_{D \in \mathbf{E} \backslash A(Q) / \mathbf{E} ; \mu(D)=\mu} \tau(D)=\sum_{D \in \mathbf{E} \backslash \mathbf{A}(\mu), \mu(D)=\mu}(\mathbf{E} D),
\end{aligned}
$$

where $\mathbf{E}=E(Q)$.

Lemma 1.5. Let $Q$ be a nonsingular even matrix of order $m$. Then the elements of $\mathcal{H}_{\text {reg }}(Q)$ of the form $(1.13),(1.14)$ satisfy the relations

$$
\tau\left[d_{1}, \ldots, d_{m}\right]^{*}=\tau\left[d_{1}, \ldots, d_{m}\right], \quad \tau[\mu]^{*}=\tau[\mu] .
$$

Proof. By (1.13) and Lemma 1.4, we have

$$
\begin{aligned}
\tau\left[d_{1}, \ldots, d_{m}\right]^{*} & =\sum_{\substack{D \in \mathbf{E} \backslash A(Q) / \mathbf{E} ; \\
\mathcal{D}(D)=\operatorname{diag}\left(d_{1}, \ldots, d_{m}\right)}} \tau\left(D^{*}\right) \\
= & \sum_{\substack{D^{*} \in \mathbf{E} \backslash A(Q) / \mathbf{E} ; \\
\mathcal{D}\left(D^{*}\right)=\operatorname{diag}\left(d_{1}, \ldots, d_{m}\right)}} \tau\left(D^{*}\right)=\tau\left[d_{1}, \ldots, d_{m}\right] .
\end{aligned}
$$

Generally speaking, the automorph class rings are noncommutative. However, in the single-class case their elements satisfy important commutation relations.

Proposition 1.6. Let $Q$ be a single-class matrix, and let $\tau(D)$ and $\tau\left(D^{\prime}\right)$ be two double cosets of $\mathcal{H}=\mathcal{H}(Q)$. Suppose that their multipliers $\mu=\mu(D)$ and $\mu^{\prime}=\mu\left(D^{\prime}\right)$ are coprime and at least one of these multipliers is relatively prime to $\operatorname{det} Q$. Then the following relations are fulfilled in the ring $\mathcal{H}$ :

$$
\begin{aligned}
& \tau(D) \tau\left(D^{\prime}\right)=\tau\left(D D^{\prime}\right), \\
& \tau(D) \tau\left(D^{\prime}\right)=\tau\left(D^{\prime}\right) \tau(D),
\end{aligned}
$$

and

$$
[a] \tau(D)=\tau(D)[a]=\tau(a D) \quad(a=1,2, \ldots)
$$

where

$$
[a]=[a]_{m}=\tau\left(a \cdot 1_{m}\right)=\tau[\underbrace{a, \ldots, a}_{m}] .
$$


Proof. We write the double cosets listed above in the form (1.6):

$$
\tau(D)=\sum_{\alpha}\left(\mathbf{E} D_{\alpha}\right), \quad \tau\left(D^{\prime}\right)=\sum_{\beta}\left(\mathbf{E} D_{\beta}^{\prime}\right), \quad \text { and } \quad \tau\left(D D^{\prime}\right)=\sum_{\gamma}\left(\mathbf{E} R_{\gamma}\right) .
$$

To prove (1.16), we must show that the products $D_{\alpha} D_{\beta}^{\prime}$ form a system of representatives for the left cosets modulo $\mathbf{E}$ contained in the double coset $\mathbf{E} D D^{\prime} \mathbf{E}$. Since

$$
\operatorname{gcd}\left(\operatorname{det} D, \operatorname{det} D^{\prime}\right)=\operatorname{gcd}\left(\mu^{m / 2},\left(\mu^{\prime}\right)^{m / 2}\right)=1,
$$

we can argue as in $[\mathrm{An}(87)$, Proposition 3.2.5] to obtain the following relation in the Hecke-Shimura ring of the group $\Lambda=S L_{m}(\mathbb{Z})$ :

$$
\begin{aligned}
(\Lambda D \Lambda)\left(\Lambda D^{\prime} \Lambda\right) & =\sum_{g \in \Lambda \backslash \Lambda D \Lambda}(\Lambda g) \sum_{h \in \Lambda \backslash \Lambda D \Lambda}(\Lambda h) \\
& =\sum_{g, h}(\Lambda g h)=\left(\Lambda D D^{\prime} \Lambda\right) .
\end{aligned}
$$

It follows that all the products $D_{\alpha} D_{\beta}^{\prime}$ are contained in the double coset $\Lambda D D^{\prime} \Lambda$, and they belong to distinct left cosets modulo $\Lambda$. In particular, they belong to distinct left cosets modulo the subgroup $\mathbf{E} \subset \Lambda$. Now, we take an arbitrary representative $R_{\gamma} \in \mathbf{E} \backslash \mathbf{E} D D^{\prime} \mathbf{E}$. By (1.20), $R_{\gamma}$ can be written in the form

$$
R_{\gamma}=g h, \quad \text { where } g \in \Lambda D \Lambda, h \in \Lambda D^{\prime} \Lambda .
$$

By (1.4), we have $R_{\gamma} \in \mathbf{A}\left(\mu \mu^{\prime}\right)$, whence $Q\left[R_{\gamma}\right]=\mu \mu^{\prime} Q$. Consequently,

$$
\mu^{-1} Q[g]=\mu^{\prime} Q\left[h^{-1}\right] .
$$

The denominators of the entries of the rational symmetric matrix on the left in (1.21) are products of primes that divide $\mu$, while the denominators on the right-hand side are products of primes that divide $\operatorname{det} h=\operatorname{det} D^{\prime}=\left(\mu^{\prime}\right)^{m / 2}$. Since $\mu$ and $\mu^{\prime}$ are relatively prime, it follows that both matrices in (1.21) are integral and have the same determinant equal to $\operatorname{det} Q$. Furthermore, since at least one of the numbers $\mu$ and $\mu^{\prime}$ is odd, both matrices are even. If, say, $\mu$ is coprime to $\operatorname{det} Q$, then, since each of the prime divisors of the divisor or the level of $Q$ must divide $\operatorname{det} Q$ (see, e.g., $\operatorname{An}(87)$, Lemma 5.1.5]), we conclude that the even matrix (1.21) has the same divisor and level, respectively, as those of $Q$. Since $Q$ is a single-class matrix, it follows that

$$
\mu^{-1} Q[g]=\mu^{\prime} Q\left[h^{-1}\right]=Q[u] \quad \text { with } u \in \Lambda,
$$

so that $Q\left[g u^{-1}\right]=\mu Q$ and $Q[u h]=\mu^{\prime} Q$. Thus, $u h \in A\left(\mu^{\prime}\right) \cap \Lambda D^{\prime} \Lambda$ and consequently, by Lemma 1.2, $u h=v D_{j}^{\prime}$ with $v \in \mathbf{E}$. Similarly, we obtain $g u^{-1} v \in A(\mu) \cap \Lambda D \Lambda$ and $g u^{-1} v=w D_{i}$ with $w \in \mathbf{E}$. Then

$$
R_{\gamma}=g h=g u^{-1} v v^{-1} u h=w D_{\alpha} D_{\beta}^{\prime} .
$$

This proves (1.16). Relation (1.17) follows from (1.16), (1.20), and the commutativity of the Hecke ring of $\Lambda$ (see $\operatorname{An}(87)$, Theorem 3.2.3]). Relations (1.18) are obvious.

Applying Proposition 1.6 to the sums (1.13), (1.14), we obtain the following.

Corollary 1.7. For a single-class matrix $Q$, the elements of $\mathcal{H}_{\mathrm{reg}}(Q)$ of the form (1.13) satisfy the relations

$$
\tau\left[d_{1}, \ldots, d_{m}\right] \tau\left[d_{1}^{\prime}, \ldots, d_{m}^{\prime}\right]=\tau\left[d_{1} d_{1}^{\prime}, \ldots, d_{m} d_{m}^{\prime}\right]
$$

if

$$
\operatorname{gcd}\left(\frac{d_{m}}{d_{1}}, \frac{d_{m}^{\prime}}{d_{1}^{\prime}}\right)=1, \quad \text { and } \quad \operatorname{gcd}\left(\frac{d_{m}}{d_{1}}, \operatorname{det} Q\right)=1 \text { or } \operatorname{gcd}\left(\frac{d_{m}^{\prime}}{d_{1}^{\prime}}, \operatorname{det} Q\right)=1
$$


and the elements (1.14) satisfy

$$
\tau(\mu) \tau\left(\mu^{\prime}\right)=\tau\left(\mu \mu^{\prime}\right)
$$

if

$$
\operatorname{gcd}\left(\mu, \mu^{\prime}\right)=1, \quad \text { and } \operatorname{gcd}(\mu, \operatorname{det} Q)=1 \text { or } \operatorname{gcd}\left(\mu^{\prime}, \operatorname{det} Q\right)=1 .
$$

Relation (1.23) implies that the elements of the form (1.14), which can be defined for all $\mu=1,2, \ldots$, belong to $\mathcal{H}=\mathcal{H}(Q)$ and satisfy (0.4); this justifies the formal Euler factorization (0.5). As to the regular Euler factors, it is known that for each integral single-class quadratic form $\mathbf{q}$ in $m=2,3$, or 4 variables with matrix $Q$, and each prime number $p$ not dividing $\operatorname{det} Q$, the following formal identities are true (we use the notation (1.13), (1.14), and (1.19)):

$$
\begin{aligned}
& \sum_{\delta=0}^{\infty} \tau\left(p^{\delta}\right) t^{\delta} \\
& \quad= \begin{cases}\left([1]-\tau(p) t+\chi_{Q}(p)[p] t^{2}\right)^{-1} & (m=2), \\
\left([1]-\left(\tau\left(p^{2}\right)-[p]\right) t^{2}+p\left[p^{2}\right] t^{4}\right)^{-1}\left([1]+[p] t^{2}\right) & (m=3), \\
\left([1]-\tau(p) t+\widetilde{\tau}\left(p^{2}\right) t^{2}-p[p] \tau(p) t^{3}+p^{2}\left[p^{2}\right] t^{4}\right)^{-1}\left([1]-\chi_{Q}(p)[p] t^{2}\right) & (m=4),\end{cases}
\end{aligned}
$$

where $\chi_{Q}$ is the character of the quadratic form q, i.e., for $p \nmid \operatorname{det} Q$ and $m=2$ we have $\chi_{Q}(p)=1$ if the congruence $\mathbf{q}(X) \equiv 0(\bmod p)$ has a nontrivial solution and $\chi_{Q}(p)=-1$ otherwise, and $\chi_{Q}(p)=\left(\frac{\operatorname{det} Q}{p}\right)$ is the Legendre symbol if $m=4$, and where

$$
\widetilde{\tau}\left(p^{2}\right)=\chi_{Q}(p) \tau\left[1, p, p, p^{2}\right]+\left(1+\chi_{Q}(p)\right) p[p]
$$

(see $[\operatorname{An}(93)]$ and $[\operatorname{An}(94)$, Theorems 1.1 and 1.3]). It follows that the local zeta-series $Z_{p}(t, Q)=Z_{p}(t, \mathbf{q})$ in these cases are of the form

$$
\begin{array}{ll}
Z_{p}(t, Q)=\left([1]-\tau(p) t+\chi_{Q}(p)[p] t^{2}\right)^{-1} & (m=2) ; \\
Z_{p}(t, Q)=\left([1]-\left(\tau\left(p^{2}\right)-[p]\right) t^{2}+p\left[p^{2}\right] t^{4}\right)^{-1} & (m=3) ; \\
Z_{p}(t, Q)=\left([1]-\tau(p) t+\widetilde{\tau}\left(p^{2}\right) t^{2}-p[p] \tau(p) t^{3}+p^{2}\left[p^{2}\right] t^{4}\right)^{-1} & (m=4) .
\end{array}
$$

It should be noted that these formulas were proved in the paper cited above without the assumption that $\mathbf{q}$ is a single-class positive definite form, but for more general automorph class rings.

The summation formulas quoted above imply new commutation relations in the regular rings of single-class forms in 2,3 , and 4 variables.

Proposition 1.8. Let $Q$ be a single-class matrix of order $m=2,3$, or 4 , and let $p$ be a prime number not dividing $\operatorname{det} Q$. Then all elements of $\mathcal{H}_{\mathrm{reg}}(Q)$ of the form $\tau\left(p^{\delta}\right)$ with $\delta=0,1,2, \ldots$ belong to the ring of polynomials over $\mathbb{Z}$ in the following elements commuting with each other: $\tau(p),[p]$ if $m=2 ; \tau\left(p^{2}\right),[p]$ if $m=3 ; \tau(p), \tau\left[1, p, p, p^{2}\right],[p]$ if $m=4$ and $\chi_{Q}(p)=1$; and $\tau\left[1, p, p, p^{2}\right],[p]$ if $m=4$ and $\chi_{Q}(p)=-1$.

Proof. The cases of $m=2$ and $m=3$ follow directly from (1.18) and formulas (1.22), (1.23). In the case where $m=4$, for the same reason, it suffices to check that the element $\tau\left[1, p, p, p^{2}\right]$ commutes with $\tau(p)$ if $\chi_{Q}(p)=1$, and that $\tau(p)=0$ if $\chi_{Q}(p)=-1$. The latter claim follows from $\left[\operatorname{An}(94)\right.$, (6.13)]. Finally, if $\chi_{Q}(p)=1$, then we can use $\operatorname{An}(94)$, (6.37)] to write

$$
\tau\left[1, p, p, p^{2}\right] \tau(p)=\tau\left[1, p, p^{2}, p^{3}\right]+(p+1)[p] \tau(p) .
$$

Thus, by Corollary 1.7 and Lemma 1.1, we obtain

$$
\tau\left[1, p, p, p^{2}\right] \tau(p)=\left(\tau\left[1, p, p, p^{2}\right] \tau(p)\right)^{*}=\tau(p)^{*} \tau\left[1, p, p, p^{2}\right]^{*}=\tau(p) \tau\left[1, p, p, p^{2}\right] .
$$




\section{$\S 2$. REPRESENTATIONS ON HARMONIC FORMS}

In this section we define linear representations of automorph class rings of integral positive definite single-class quadratic forms on spaces of harmonic polynomials and consider the question concerning the existence of eigenfunctions for the operators of these representations.

We recall that a polynomial $P_{0}=P_{0}(X)$ with complex coefficients, where $X=\left(x_{i j}\right)$ is an $(m \times n)$-matrix of variables, is called a harmonic form of order $m$, genus $n$, and weight $k$ if it is a harmonic function in $m n$ variables $x_{i j}$ in the sense that

$$
\Delta P_{0}=\sum_{i, j} \frac{\partial^{2} P_{0}}{\partial x_{i j}^{2}}=0
$$

and satisfies the condition

$$
P_{0}(X A)=(\operatorname{det} A)^{k} P_{0}(X) \text { for every matrix } A \in G L_{n}(\mathbb{C}) .
$$

This definition implies that, for every harmonic form $P_{0}$ of order $m$, genus $n$, and weight $k$ and every matrix $U$ of order $m$ in the real orthogonal group $O_{m}(\mathbb{R})$, the polynomial $P_{0}(U X)$ is again a harmonic form of order $m$, genus $n$, and weight $k$. In this sense the definition of the harmonic forms is related to the quadratic form $\mathbf{q}_{0}=x_{1}^{2}+\cdots+x_{m}^{2}$ with the matrix $Q_{0}=2 \cdot 1_{m}$, for which the group of real automorphisms is $O_{m}(\mathbb{R})$.

Now, we are going to introduce the harmonic forms that are related in a similar manner to an arbitrary real positive definite quadratic form (1.1) in $m$ variables with matrix $Q$. Since the matrix $Q$ is positive definite, there is a real matrix $S$ such that $Q=2{ }^{t} S S$, and for a harmonic form $P_{0}$ of order $m$, genus $n$, and weight $k$ we define a harmonic form $P=P_{Q}(X)$ of genus $n$ and weight $k$ with respect to the quadratic form with matrix $Q$ (or simply with respect to $Q$ ) by putting

$$
P(X)=P_{Q}(X)=\left(P_{0} \mid S\right)(X)=P_{0}(S X) .
$$

By (2.2), obviously, this polynomial satisfies

$$
P(X A)=(\operatorname{det} A)^{k} P(X) \text { for every matrix } A \in G L_{n}(\mathbb{C}),
$$

and for every matrix

$$
U \in O(Q, \mathbb{R})=\left\{U \in \mathbb{R}_{m}^{m} \mid Q[U]=Q\right\}=S^{-1} O_{m}(\mathbb{R}) S
$$

the polynomial

$$
(P \mid U)(X)=P(U X)=P_{0}(S U X)=\left(P_{0} \mid S U S^{-1}\right)(S X)
$$

is again a harmonic form of genus $n$ and weight $k$ with respect to $Q$. Clearly, the set $\mathcal{P}_{k}^{n}(Q)$ of all harmonic forms of genus $n$ and weight $k$ with respect to $Q$ is a linear space over $\mathbb{C}$. By $(2.4)$, each polynomial in $\mathcal{P}_{k}^{n}(Q)$ is homogeneous of degree $n k$. Thus, the space $\mathcal{P}_{k}^{n}(Q)$ is finite-dimensional.

The general linear group $G L_{m}(\mathbb{R})$ acts on the functions $P: \mathbb{R}_{n}^{m} \mapsto \mathbb{C}$ by linear transformations of variables,

$$
U \mapsto \mid U: P(X) \mapsto(P \mid U)(X)=P(U X) \quad\left(U \in G L_{m}(\mathbb{R})\right),
$$

these operators preserve the polynomials satisfying (2.2), and we have

$$
|U| V=\mid U V \quad\left(U, V \in G L_{m}(\mathbb{R})\right)
$$

Lemma 2.1. Each of the operators $\mid U$ with $U \in G L_{m}(\mathbb{R})$ maps the space $\mathcal{P}_{k}^{n}(Q)$ bijectively onto $\mathcal{P}_{k}^{n}(Q[U])$. 
Proof. If $S$ satisfies $2{ }^{t} S S=Q$, then $Q[U]=2{ }^{t}(S U)(S U)$. Thus, by definition,

$$
P_{Q[U]}(X)=P_{0}(S U X)=\left(P_{Q} \mid U\right)(X),
$$

where $P_{0}=P_{2 \cdot 1_{m}}(X) \in \mathcal{P}_{k}^{n}\left(Q_{0}\right)$ is a harmonic form of genus $n$ and weight $k$.

Now we define a (Hermitian) scalar product of every two (say, continuous) functions $f, g: \mathbb{R}_{n}^{m} \mapsto \mathbb{C}$ relative to the matrix $Q$ of a positive definite quadratic form in $m$ variables:

$$
(f, g)=(f, g)_{Q}=(\operatorname{det} Q)^{\frac{n}{2}} \int_{\frac{1}{2} Q[X] \leq 1_{n}} f(X) \overline{g(X)} d X,
$$

where $d X=d\left(x_{i j}\right)=\prod_{i, j} d x_{i j}$ is the Euclidean volume element in $\mathbb{R}_{n}^{m}$, and the inequality $A \leq B$ for two real symmetric matrices of the same order means that the matrix $B-A$ is positive semidefinite.

Lemma 2.2. For any matrix $U \in G L_{m}(\mathbb{R})$ and any functions $f, g: \mathbb{R}_{n}^{m} \mapsto \mathbb{C}$, the scalar product (2.7) satisfies the relation

$$
(f|U, g| U)_{Q[U]}=(f, g)_{Q}
$$

where $\mid U$ is the operator (2.5). In particular,

$$
(f \mid U, g)_{Q}=\left(f, g \mid U^{-1}\right)_{Q} \quad \text { if } U \in O(Q, \mathbb{R}) .
$$

Proof. By the change of variables $X \mapsto Y=U X$, we obtain

$$
\begin{aligned}
(f|U, g| U)_{Q[U]} & =|\operatorname{det} U|^{n}(\operatorname{det} Q)^{\frac{n}{2}} \int_{\frac{1}{2} Q[U X] \leq 1_{n}} f(U X) \overline{g(U X)} d X \\
& =(\operatorname{det} Q)^{\frac{n}{2}} \int_{\frac{1}{2} Q[Y] \leq 1_{n}} f(Y) \overline{g(Y)} d Y .
\end{aligned}
$$

The lemma follows.

The following proposition describes the spaces of harmonic forms of genus $n$ and weight $k$ with respect to positive definite quadratic forms.

Proposition 2.3. The space of harmonic forms $\mathcal{P}_{k}^{n}(Q)$ relative to the matrix $Q$ of a positive definite quadratic form in $m$ variables is spanned over $\mathbb{C}$ by the polynomials

$$
P(X)=\operatorname{det}\left({ }^{t} \Omega Q X\right)^{k},
$$

where $\Omega$ is a matrix in $\mathbb{C}_{n}^{m}$ satisfying ${ }^{t} \Omega Q \Omega=0$ if $k>1$.

Proof. If $S$ is a real matrix satisfying $Q=2{ }^{t} S S$, then $P(X)=P_{0}(S X)$, where $P_{0} \in$ $P_{k}^{n}\left(Q_{0}\right)$ with $Q_{0}=2 \cdot 1_{m}$. By a consequence of the Kashiwara-Verne theory (see $[\mathrm{KV}(78)]$ ) observed by Freitag in $\operatorname{Fr}(91)$, Proposition 6.20], our proposition is true for $Q=Q_{0}$. Then it is also true for $Q={ }^{t} S Q_{0} S$, because ${ }^{t} \Omega Q \Omega=0$ means that ${ }^{t}(S \Omega) Q_{0}(S \Omega)=0$. (For a simple proof for $n=1$, see $\operatorname{Ogg}(69)$, Chapter VI].)

Let $\mathcal{I}_{k}^{n}(Q)$ be the subspace of all polynomials in $\mathcal{P}_{k}^{n}(Q)$ that are invariant with respect to all operators $\mid U$ of the form (2.5) with $U \in E(Q)$, i.e.,

$$
\mathcal{I}_{k}^{n}(Q)=\left\{P \in \mathcal{P}_{k}^{n}(Q) \mid P(U X)=P(X) \text { for all } U \in E(Q)\right\} .
$$

With each element

$$
\tau=\sum_{\alpha} a_{\alpha}\left(E(Q) D_{\alpha}\right) \in \mathcal{H}=\mathcal{H}(Q),
$$

we associate the Hecke operator $\mid \tau$ on $\mathcal{I}_{k}^{n}(Q)$ given by

$$
|\tau: P \mapsto P| \tau=\sum_{\alpha} a_{\alpha} P \mid D_{\alpha} \quad\left(P \in \mathcal{I}_{k}^{n}(Q)\right),
$$


where the $\mid D_{\alpha}$ are the operators (2.5). Relation (2.6) and the definitions of the space $\mathcal{I}_{k}^{n}(Q)$ and the ring $\mathcal{H}$ show that each Hecke operator is independent of the choice of representatives in the corresponding left cosets and maps this space into itself. Moreover, by (2.6) and the definition of multiplication in $\mathcal{H}$, any product of the elements in $\mathcal{H}$ goes to the product of corresponding operators. Thus, the mapping $\tau \mapsto \mid \tau$ is a linear representation of the ring $\mathcal{H}(Q)$ on the space $\mathcal{I}_{k}^{n}(Q)$.

Theorem 2.4. For every even positive definite matrix $Q$ and every element $\tau$ of the regular automorph class ring $\mathcal{H}_{\text {reg }}=\mathcal{H}_{\text {reg }}(Q)$, the Hecke operators $\mid \tau$ and $\mid \tau^{*}$ on the space $\mathcal{I}_{k}^{n}(Q)$ (the star stands for the antiautomorphism (1.8)) are conjugate with respect to the scalar product (2.7), i.e.,

$$
\left(P \mid \tau, P^{\prime}\right)_{Q}=\left(P, P^{\prime} \mid \tau^{*}\right)_{Q} \quad\left(P, P^{\prime} \in \mathcal{I}_{k}^{n}(Q), \tau \in \mathcal{H}(Q)\right) .
$$

Proof. It suffices to prove (2.14) in the case where $\tau=\tau(D)$ is a double coset of the form (1.6) with $D$ belonging to a set $\mathbf{A}(\mu)=A(\mu, Q)$ of the form (1.3) with a positive integer $\mu$ coprime to $\operatorname{det} Q$. Clearly, if $D^{\prime} \in \mathbf{E} D \mathbf{E} \in \mathbf{A}(\mu)$, where $\mathbf{E}=E(Q)$, then $(\sqrt{\mu})^{-1} D^{\prime} \in O(Q, \mathbb{R})$, and, by $(2.4)$, for every polynomial $R \in \mathcal{P}_{k}^{n}(Q)$ we can write

$$
R\left|D^{\prime}=P\right| D^{\prime}\left((\sqrt{\mu})^{-1} \sqrt{\mu} \cdot 1_{m}\right)=\mu^{m k / 2} R \mid(\sqrt{\mu})^{-1} D^{\prime} .
$$

Hence, by Lemma 2.2 and the definition of the Hecke operators, we have

$$
\begin{aligned}
(P \mid \tau & \left.(D), P^{\prime}\right)=\left(P \mid \sum_{D^{\prime} \in \mathbf{E} \backslash \mathbf{E} D \mathbf{E}}\left(\mathbf{E} D^{\prime}\right), P^{\prime}\right)=\sum_{D^{\prime} \in \mathbf{E} \backslash \mathbf{E} D \mathbf{E}}\left(P \mid D^{\prime}, P^{\prime}\right) \\
= & \sum_{D^{\prime} \in \mathbf{E} \backslash \mathbf{E} D \mathbf{E}} \mu^{n k / 2}\left(P \mid(\sqrt{\mu})^{-1} D^{\prime}, P^{\prime}\right)=\sum_{D^{\prime} \in \mathbf{E} \backslash \mathbf{E} D \mathbf{E}} \mu^{n k / 2}\left(P, P^{\prime} \mid \sqrt{\mu}\left(D^{\prime}\right)^{-1}\right) \\
= & \sum_{D^{\prime} \in \mathbf{E} \backslash \mathbf{E} D \mathbf{E}}\left(P, P^{\prime} \mid\left(D^{\prime}\right)^{*}\right) .
\end{aligned}
$$

Since $\left\{D^{\prime}\right\}$ is a system of representatives for the left cosets modulo $\mathbf{E}$ in the double coset $\mathbf{E} D \mathbf{E}$, Lemma 1.1 shows that $\left\{\left(D^{\prime}\right)^{*}\right\}$ is a system of representatives for the right cosets modulo $\mathbf{E}$ in $\mathbf{E} D^{*} \mathbf{E}$. If we can choose the system $\left\{D^{\prime}\right\}$ so that it is a set of common representatives both for the left and right cosets of $\mathbf{E} D \mathbf{E}$ modulo $\mathbf{E}$, then the set $\left\{\left(D^{\prime}\right)^{*}\right\}$ will also be a set of common representatives for the left and the right cosets of $\mathbf{E} D^{*} \mathbf{E}$ modulo $\mathbf{E}$, which would imply that the right-hand side of (2.16) can be written in the form

$$
\sum_{\left(D^{\prime}\right)^{*} \in \mathbf{E} \backslash \mathbf{E} D^{*} \mathbf{E}}\left(P, P^{\prime} \mid\left(D^{\prime}\right)^{*}\right)=\left(P, P^{\prime} \mid \tau\left(D^{*}\right)\right)=\left(P, P^{\prime} \mid \tau(D)^{*}\right),
$$

as desired.

Since each left coset of $\mathbf{E} D \mathbf{E}$ modulo $\mathbf{E}$ meets each right coset, in order to prove the existence of a common system of representatives both for the right and the left cosets it suffices to check that the number of left cosets is equal to the number of right cosets. By An(87), Lemma 3.1.2], the number of left cosets modulo $\mathbf{E}$ in the double coset $\mathbf{E} D \mathbf{E}$ is equal to the index $\nu=\left[\mathbf{E}: \mathbf{E} \cap D^{-1} \mathbf{E} D\right]$. Similarly, the number of right cosets modulo $\mathbf{E}$ in $\mathbf{E} D \mathbf{E}$ is equal to the index $\nu^{\prime}=\left[\mathbf{E}: \mathbf{E} \cap D \mathbf{E} D^{-1}\right]$. Since the matrix $Q$ is positive definite, the group $\mathbf{E}$ is finite. Clearly, the finite subgroups $\mathbf{E} \cap D^{-1} \mathbf{E} D$ and $\mathbf{E} \cap D \mathbf{E} D^{-1}$ are conjugate in $O(Q, \mathbb{R})$, so that they are of the same order. Thus, $\nu=\nu^{\prime}$.

Proposition 2.5. For a positive definite even matrix $Q$, the Hecke operators on the space $\mathcal{I}_{k}^{n}(Q)$ corresponding to every system of mutually commuting elements $\tau \in \mathcal{H}_{\mathrm{reg}}(Q)$ with $\tau^{*}=\tau$ can be diagonalized simultaneously on each invariant subspace. 
Proof. By Theorem 2.3, the Hecke operators $\mid \tau$ with $\tau$ satisfying $\tau^{*}=\tau$ are selfadjoint with respect to the Hermitian scalar product (2.7). By a well-known theorem of linear algebra, any family of mutually commuting selfadjoint linear operators on a finitedimensional Hilbert space can be diagonalized simultaneously.

\section{§3. Action of Hecke operators on theta-Series WITH HARMONIC COEFFICIENTS}

Given an integral positive definite quadratic form (1.1) with matrix $Q$ and a harmonic form $P(X) \in \mathcal{P}_{k}^{n}(Q)$ of weight $k$ and genus $n$ with respect to $Q$, we introduce the theta-series of $Q$ of genus $n$ with the harmonic form $P$ by

$$
\theta(Z ; P, Q)=\sum_{N \in \mathbb{Z}_{n}^{m}} P(N) e^{\pi i \sigma(Q[N] Z)}
$$

where the variable $Z$ belongs to the upper half-plane $\mathbb{H}_{n}$ of genus $n$,

$$
\mathbb{H}_{n}=\left\{Z=X+\left.i Y \in \mathbb{C}_{n}^{n}\right|^{t} Z=Z, Y>0\right\},
$$

and $\sigma(A)$ denotes the trace of a square matrix $A$. Obviously, this series converges absolutely and uniformly on the compact subsets of $\mathbb{H}_{n}$, thus giving a holomorphic function in $n(n+1) / 2$ complex variables. The Fourier expansion of the theta-series

$$
\theta(Z ; P, Q)=\sum_{A \in \mathbb{E}_{n}, A \geq 0} r(A ; P, Q) e^{\pi i \sigma(A Z)}
$$

has constant Fourier coefficients

$$
r(A ; P, Q)=\sum_{N \in \mathbb{Z}_{n}^{m}, Q[N]=A} P(N) .
$$

Replacing $N$ by $U N$ with $U \in \Lambda^{m}$, we get the identity

$$
\theta\left(Z ; P \mid U,{ }^{t} U Q U\right)=\theta(Z ; P, Q),
$$

where $P \mid U$ is defined by (2.5); in particular,

$$
\theta(Z ; P \mid U, Q)=\theta(Z ; P, Q), \quad U \in E(Q) .
$$

After the replacement, if necessary, of the polynomial $P$ by its average

$$
\#(E(Q))^{-1} \sum_{U \in E(Q)} P \mid U
$$

over the unit group $E(Q)$, which does not change the theta-series, without loss of generality we may assume that $P \in \mathcal{I}_{k}^{n}(Q)$.

By $\operatorname{AM}(75)]$ (see also $[\operatorname{An}(95)]$ ), if $m$ is even, then the theta-series (3.1) belongs to the space $\mathfrak{M}_{m / 2+k}^{n}\left(q, \chi_{Q}\right)$ of modular forms of weight $m / 2+k$ for the group

$$
\Gamma_{0}^{n}(q)=\left\{M=\left(\begin{array}{ll}
A & B \\
C & D
\end{array}\right) \in \operatorname{Sp}_{n}(\mathbb{Z}) \mid C \equiv 0(\bmod q)\right\},
$$

where $q$ is the level of $Q$, with the Dirichlet character $\chi_{Q}$ modulo $q$ satisfying the conditions $\chi_{Q}(-1)=(-1)^{m / 2}$ and

$$
\chi_{Q}(p)=\left(\frac{(-1)^{m / 2} \operatorname{det} Q}{p}\right) \quad(\text { the Legendre symbol) }
$$

if $p$ is an odd prime number not dividing $q$. In particular, for the theta-series we have

$$
\operatorname{det}(C Z+D)^{-(m / 2+k)} \theta\left((A Z+B)(C Z+D)^{-1} ; P, Q\right)=\chi_{Q}(\operatorname{det} D) \theta(Z ; P, Q)
$$

for every matrix $\left(\begin{array}{ll}A & B \\ C & D\end{array}\right) \in \Gamma_{0}^{n}(q)$. 
Following the general pattern of the theory of Hecke operators on spaces of modular forms (see, e.g., $\operatorname{An}(87)$, Chapter 4], or $\operatorname{An}(96), \S 2]$ ), now we recall the basic definitions and the simplest properties of (regular) Hecke operators on the spaces $\mathfrak{M}_{r}^{n}(q, \chi)$ of modular forms of integral weight $r$ and character $\chi$ for the group $\Gamma_{0}^{n}(q)$. Let

$$
\mathcal{H}_{0}^{n}(q)=\mathcal{H}\left(\Gamma_{0}^{n}(q), \Sigma_{0}^{n}(q)\right)
$$

denote the Hecke-Shimura ring of the semigroup

$$
\begin{aligned}
\Sigma_{0}^{n}(q)=\{ & M=\left(\begin{array}{ll}
A & B \\
C & D
\end{array}\right) \in \mathbb{Z}_{2 n}^{2 n} \mid \\
\left.{ }^{t} M J_{n} M=\mu(M) J_{n}, \mu(M)>0, \operatorname{gcd}(\operatorname{det} M, q)=1, C \equiv 0(\bmod q)\right\} & \left(J_{n}=\left(\begin{array}{cc}
0 & 1_{n} \\
-1_{n} & 0
\end{array}\right)\right)
\end{aligned}
$$

relative to the group $\Gamma_{0}^{n}(q)$ (over $\mathbb{C}$ ). The ring $\mathcal{H}_{0}^{n}(q)$ is generated over $\mathbb{C}$ by the mutually commuting algebraically independent elements

$$
\left\{\begin{array}{l}
T^{n}(p)=(\operatorname{diag}(\underbrace{1, \ldots, 1}_{n}, \underbrace{p, \ldots, p}_{n}))_{\Gamma_{0}^{n}(q)}, \\
T_{j}^{n}\left(p^{2}\right)=\left(\operatorname{diag}(\underbrace{1, \ldots, 1}_{n-j}, \underbrace{p, \ldots, p}_{j}, \underbrace{p^{2}, \ldots, p^{2}}_{n-j}, \underbrace{p, \ldots, p}_{j})\right)_{\Gamma_{0}^{n}(q)}^{p(1 \leq j \leq n),}
\end{array}\right.
$$

where $p$ runs over all prime numbers not dividing $q$, and

$$
(M)=(M)_{\Gamma}=\sum_{M^{\prime} \in \Gamma \backslash \Gamma M \Gamma}\left(\Gamma M^{\prime}\right) \quad\left(\Gamma=\Gamma_{0}^{n}(q), M \in \Sigma_{0}^{n}(q)\right)
$$

is the double coset of $M$ modulo $\Gamma_{0}^{n}(q)$ (see $\operatorname{An}(87)$, Theorem 3.3.23]).

For

$$
T=\sum_{i} c_{i}\left(\Gamma_{0}^{n}(q) M_{i}\right) \in \mathcal{H}_{0}^{n}(q),
$$

the Hecke operator $|T=|_{r, \chi} T$ on the space $\mathfrak{M}_{r}^{n}(q, \chi)$ can be defined by

$$
F\left|T=\sum_{i} c_{i} F\right|_{r, \chi} M_{i} \quad\left(F \in \mathfrak{M}_{r}^{n}(q, \chi)\right)
$$

where

$$
\begin{aligned}
\left.F\right|_{r, \chi}\left(\begin{array}{cc}
A & B \\
C & D
\end{array}\right)=\chi(\operatorname{det} A) \operatorname{det}(C Z+D)^{-r} F\left((A Z+B)(C Z+D)^{-1}\right) \\
\left(\left(\begin{array}{ll}
A & B \\
C & D
\end{array}\right) \in \Sigma_{0}^{n}(q)\right)
\end{aligned}
$$

are the Petersson operators. The Hecke operators map the space $\mathfrak{M}_{r}^{n}(q, \chi)$ into itself and are independent of the choice of representatives $M_{i} \in \Gamma_{0}^{n}(q) M_{i}$.

Quite often, Hecke operators map theta-series to linear combinations of similar thetaseries. An easy modification of a particular case of $\mathrm{An}(96)$, Theorem 4.1] can be formulated as follows. Let $Q$ be an even positive definite single-class matrix of even order $m$, and let $q$ be the level of $Q$. Suppose that a double coset $(M)=(M)_{\Gamma_{0}^{n}(q)} \in \mathcal{H}_{0}^{n}(q)$ of the form (3.6), where $n \leq m$, belongs to the image of the $\operatorname{ring} \mathcal{H}_{0}^{m}(q)$ under the Zharkovskaya map

$$
\Psi^{m, n}=\Psi_{Q}^{m, n}=\Psi_{m / 2, \chi_{Q}}: \mathcal{H}_{0}^{m}(q) \mapsto \mathcal{H}_{0}^{n}(q)
$$


(see $\operatorname{An}(87), \S 4.2 .4]$ and $[\operatorname{An}(96), \S 3])$. Then the image of the theta-series (3.1) of genus $n$ with a harmonic form $P \in \mathcal{I}_{k}^{n}(Q)$ under the action of the Hecke operator corresponding to a double coset $(M)$ can be written in the form

$$
\theta(Z ; P, Q) \mid(M)=\sum_{D \in A(Q, \mu) / E(Q)} I\left(D, Q, \Psi^{n, m}(M)\right) \theta\left(Z ; P \mid \mu^{-1} D, Q\right)
$$

with certain constant coefficients $I\left(D, Q, \Psi^{n, m}(M)\right)$, where $\mu=\mu(M), A(Q, \mu)$ is defined by (1.3), $E(Q)=A(Q, 1), \Psi^{n, m}(M) \in \mathcal{H}_{0}^{m}(q)$ is an inverse image of $(M)$ under the map $\Psi^{m, n}$, and the operators $P \mapsto P \mid U$ are defined by (2.5).

The following formulas contain all that is known at present about computation of the coefficients in (3.9) for the action of the generators (3.5): for each prime number $p$ not dividing $q$, we have

$$
I\left(D, Q, T^{m}(p)\right)= \begin{cases}p^{m / 2} \prod_{j=1}^{m / 2}\left(1+\chi_{Q}(p) p^{-j}\right) & \text { if } D \in \Lambda D_{m / 2}^{m}(p) \Lambda, \\ 0 & \text { otherwise }\end{cases}
$$

where $\Lambda=\Lambda^{m}=G L_{m}(\mathbb{Z}), D_{m / 2}^{m}(p)=\operatorname{diag}(\underbrace{1, \ldots, 1}_{m / 2}, \underbrace{p, \ldots, p}_{m / 2})$;

$$
I\left(D, Q, T_{m-1}^{m}\left(p^{2}\right)\right)= \begin{cases}\chi_{Q}(p) p^{\left(2+m-m^{2}\right) / 2} & \text { if } D \in \Lambda D_{m-2,1}^{m}(p) \Lambda \\ \alpha_{m}(p) & \text { if } D \in \Lambda\left(p 1_{m}\right) \\ 0 & \text { otherwise }\end{cases}
$$

where $D_{m-2,1}^{m}(p)=\operatorname{diag}(1, \underbrace{p, \ldots, p}_{m-2}, p^{2})$, and

$$
\begin{aligned}
\alpha_{m}(p) & =\chi_{Q}(p) p^{\left(2+m-m^{2}\right) / 2} \frac{\left(p^{m}-1\right)}{p-1}+p^{-m^{2} / 2}\left(\chi_{Q}(p) p^{m / 2}-1\right), \\
I\left(D, Q,\langle p\rangle_{m}\right) & = \begin{cases}p^{-m^{2} / 2} & \text { if } D \in \Lambda\left(p 1_{m}\right), \\
0 & \text { otherwise, }\end{cases}
\end{aligned}
$$

where for brevity we have set

$$
T_{n}^{n}\left(p^{2}\right)=\left(p \cdot 1_{2 n}\right)_{\Gamma_{0}^{n}(q)}=\langle p\rangle_{n} .
$$

(In [An(91), formula (2.19) and Lemma 5.1] the sums $\gamma(Q, D, T)$ similar to the coefficients $I(D, Q, T)$ were defined and computed for $T=T^{m}(p)$. In $[\operatorname{An}(93)$, $\S 2]$ the sums $\gamma(Q, D, T)$ were, in fact, computed for $T=\langle p\rangle_{m}=T_{m}^{m}\left(p^{2}\right)=\left(p 1_{2 m}\right)_{\Gamma_{0}^{m}(q)}$ and $T=T_{m-1}^{m}\left(p^{2}\right)$; see also $\operatorname{An}(87)$, Lemma 3.3.32] for the presentation of $T_{m-1}^{m}\left(p^{2}\right)$ used in $\mathrm{An}(93)$. The definition of these sums shows immediately that $I(D, Q,(M))=$ $\chi_{Q}(\mu)^{m} \mu^{m / 2} \gamma\left(Q, \mu D^{-1},(M)\right)=\mu^{m / 2} \gamma\left(Q, \mu D^{-1}, T\right)$, where $\mu=\mu(M)$.)

In particular, formulas (3.10)-(3.12) imply that, for the elements

$$
T=T^{m}(p), \quad\langle p\rangle_{m}=T_{m}^{m}\left(p^{2}\right), \quad \text { and } \quad T_{m-1}^{m}\left(p^{2}\right)
$$

with primes $p$ not dividing $\operatorname{det} Q$, the coefficients $I(D, Q, T)$ as functions of $D$ depend only on the double coset $\Lambda D \Lambda$. Therefore, if $\Psi^{n, m}(M)$ is a linear combination of elements 
(3.13), then, under the same assumptions, formula (3.9) can be rewritten as

$$
\begin{aligned}
\theta(Z ; P, Q) \mid(M) & =\mu^{-n k} \sum_{D \in E(Q) \backslash A(Q, \mu)} I\left(D, Q, \Psi^{n, m}(M)\right) \theta(Z ; P \mid D, Q) \\
& =\mu^{-n k} \sum_{\substack{d_{1}|\cdots| d_{m} ; \\
d_{i} d_{m-i+1}=\mu}} I\left(\operatorname{diag}\left(d_{1}, \ldots, d_{m}\right), Q, \Psi^{n, m}(M)\right) \theta\left(Z ; P \mid \tau\left[d_{1}, \ldots, d_{m}\right], Q\right) .
\end{aligned}
$$

Here we have also used (2.4), (3.3), the notation (1.13), the fact that each double coset $E(Q) D E(Q) \in A(Q, \mu)$ contains a common system of representatives both for the left and the right cosets modulo $E(Q)$ (see the end of the proof of Theorem 2.4), and the definition of the Hecke operators (2.13).

Note that, in particular, formulas (3.10)-(3.12) determine the sums $I(D, Q, T)$ for all generators of the rings $\mathcal{H}_{0}^{1}(q)$ and $\mathcal{H}_{0}^{2}(q)$, provided that we can explicitly express inverse images $\Psi^{n, m}((M))$ of the generators (3.5) for $n=1,2$ in terms of the elements (3.13). For this, first we consider the action of the Zharkovskaya map on the corresponding elements.

Lemma 3.1. The following formulas are true for the action of the Zharkovskaya map $\Psi=\Psi_{r, \chi}^{n, n-1}: \mathcal{H}_{0}^{n}(q) \mapsto \mathcal{H}_{0}^{n-1}(q)$ on some of the elements (3.5) for $n>1$ and each prime number $p$ not dividing $q$ :

$$
\begin{aligned}
\Psi^{n, n-1}\left(T^{n}(p)\right) & =\left(1+\bar{\chi}(p) p^{n-r}\right) T^{n-1}(p) ; \\
\Psi^{n, n-1}\left(\langle p\rangle_{n}\right) & =\bar{\chi}(p) p^{-r}\langle p\rangle_{n-1} ; \\
\Psi^{n, n-1}\left(T_{n-1}^{n}\left(p^{2}\right)\right) & =\bar{\chi}(p) p^{1-r} T_{n-2}^{n-1}\left(p^{2}\right)+b_{n}(p)\langle p\rangle_{n-1},
\end{aligned}
$$

where $b_{n}(p)=b_{n, r, \chi}(p)=\bar{\chi}\left(p^{2}\right) p^{2 n-2 r}+\bar{\chi}(p)(p-1) p^{-r}+1$.

Proof. The action of the Zharkovskaya map corresponding to the action of the Hecke operators on the spaces $\mathfrak{M}_{r}^{n}(q, \chi)$ was calculated in [An(87), Subsection 4.2.4]. However, applying the results of this calculation, we must take into account the fact that the Hecke operators defined in $\mathrm{An}(87),(2.4 .11)$, and (2.4.12)] have normalization different from that used here and in $[\mathrm{An}(96)$, formulas (1.10) with $l=0,(2.13),(2.14)$, and (2.20) with $Q=H$ and $P=0]$ and differ from the latter operators by the factor $\chi\left(\mu^{n}\right) \mu^{n r-n(n+1) / 2}$ on the homogeneous elements of the multiplier $\mu$.

The formula (3.15) follows from Propositions 4.2.17 and 4.2.18 and formula (4.2.80) in $\mathrm{An}(87)$ applied to the Hecke operators $\|_{r} \chi\left(p^{n}\right) p^{n r-n(n+1) / 2} T^{n}(p)$ (note the difference in normalization mentioned above). Formula (3.16) follows by similar arguments from $\mathrm{An}(87)$, Lemma 3.3.34]. As to formula (3.17), the situation is slightly more complicated. First, by using $\mathrm{An}(87)$, factorization (3.5.69) of Theorem 3.5.23, formulas (3.5.34), (3.4.15), (3.5.33), and (3.3.61)], we get the relation

$$
T_{n-1}^{n}\left(p^{2}\right)=-p^{n}\langle p\rangle_{n} \mathbf{r}_{1}^{n}(p)+\left(p^{n}-1\right)\langle p\rangle_{n},
$$

where $\mathbf{r}_{1}^{n}(p)$ is the first coefficient of the Rankin $p$-polynomial $R_{p}^{n}(v)$ defined in $\operatorname{An}(87)$, formulas (3.5.15) and (3.5.16)]. Since $\mu\left(\mathbf{r}_{1}^{n}(p)\right)=1$, from $[\operatorname{An}(87)$, Theorem 4.2.18 and relation $(4.2 .82)]$ it follows that

$$
\Psi^{n, n-1}\left(\mathbf{r}_{1}^{n}(p)\right)=\mathbf{r}_{1}^{n-1}(p)-\bar{\chi}(p) p^{n-r}-\chi(p) p^{r-n} .
$$

Since the Zharkovskaya map is a ring homomorphism, formula (3.17) follows from (3.18), (3.16), (3.19) by an easy computation. 
Applying formulas (3.15) repeatedly for the images of $T^{m}(p), \ldots, T^{n}(p)$ with $r=m / 2$, $\chi=\chi_{Q}$, and $n<m$, we obtain

$$
\Psi^{m, n}\left(T^{m}(p)\right)=\gamma_{n}^{m}(p) T^{n}(p), \quad \text { where } \gamma_{n}^{m}(p)=\left\{\prod_{i=0}^{m-n-1}\left(1+\chi_{Q}(p) p^{m / 2-i}\right)\right\} .
$$

The factor $\gamma_{n}^{m}(p)$ is equal to 0 if and only if $m / 2 \leq m-n-1$, i.e., $n \leq m / 2-1$, and $\chi_{Q}(p)=-1$. Hence,

$$
\Psi^{n, m}\left(T^{n}(p)\right)=\gamma_{n}^{m}(p)^{-1} T^{m}(p) \quad \text { unless } n \leq m / 2-1 \text { and } \chi_{Q}(p)=-1 .
$$

Similarly, by (3.16), $\Psi^{m, n}\left(\langle p\rangle_{m}\right)=\left(\chi_{Q}(p) p^{-m / 2}\right)^{m-n}\langle p\rangle_{n}$, whence

$$
\Psi^{n, m}\left(\langle p\rangle_{n}\right)=\chi_{Q}(p)^{n} p^{m(m-n) / 2}\langle p\rangle_{m}
$$

because $\chi_{Q}(p)= \pm 1$. By an easy induction, for $2 \leq n<m$, from (3.16) and (3.17) we deduce the relations

$$
\Psi^{m, n}\left(T_{m-1}^{m}\left(p^{2}\right)\right)=(a p)^{m-n} T_{n-1}^{n}\left(p^{2}\right)+a^{m-n-1}\left(\sum_{i=0}^{m-n-1} p^{i} b_{m-i}(p)\right)\langle p\rangle_{n},
$$

where $a=\chi_{Q}(p) p^{-m / 2}$. This relation and (3.21) imply that we can take

$$
\begin{aligned}
\Psi^{n, m}\left(T_{n-1}^{n}\left(p^{2}\right)\right)= & \chi_{Q}(p)^{n} p^{(m-n)(m-2) / 2} T_{m-1}^{m}\left(p^{2}\right) \\
& -\chi_{Q}(p)^{n+1} p^{\left(m^{2}-m n-m+2 n\right) / 2}\left(\sum_{i=0}^{m-n-1} p^{i} b_{m-i}(p)\right)\langle p\rangle_{m}
\end{aligned}
$$

(note that $\chi_{Q}(p)= \pm 1$ and $m$ is even), whence, in particular,

$$
\begin{aligned}
\Psi^{2,4}\left(T_{1}^{2}\left(p^{2}\right)\right) & =p^{2} T_{3}^{4}\left(p^{2}\right)-\chi_{Q}(p) p^{4}\left(b_{4}(p)+p b_{3}(p)\right)\langle p\rangle_{4} \\
& =p^{2} T_{3}^{4}\left(p^{2}\right)-\chi_{Q}(p) p^{2}\left(p^{6}+p^{5}+p^{3}+p^{2}+\chi_{Q}(p)\left(p^{2}-1\right)\right)\langle p\rangle_{4} .
\end{aligned}
$$

Now we turn to formulas for the action on theta-series of the Hecke operators corresponding to certain coefficients of the spinor $p$-polynomials

$$
\mathbf{Q}_{p}^{n}(t)=\sum_{j=0}^{2^{n}}(-1)^{j} \mathbf{q}_{j}^{n}(p) t^{j}
$$

over $p$-subrings of the rings $\mathcal{H}_{0}^{n}(q)$ for prime $p$ not dividing $q$ (see, e.g., $\operatorname{An}(87)$, (3.3.78)]). These polynomials are of considerable interest because the substitution $t=\psi(p) p^{-s}$ with a Dirichlet character $\psi$ and the replacement of the coefficients by the eigenvalues $\Lambda\left(\mathbf{q}_{j}^{n}(p)\right)$ of the corresponding Hecke operators acting on an eigenfunction $F \in \mathfrak{M}_{r}^{n}(q, \chi)$ lead to the denominators

$$
\mathbf{Q}_{p}\left(\psi(p) p^{-s}, \Lambda\right)=\sum_{j=0}^{2^{n}}(-1)^{j} \Lambda\left(\mathbf{q}_{j}^{n}(p)\right) p^{-s j}
$$

of the $p$-factor of the regular zeta function with character $\psi$,

$$
Z_{F}(s, \psi)=\prod_{p \nmid q} \mathbf{Q}_{p}\left(\psi(p) p^{-s}, \Lambda\right)^{-1},
$$

corresponding to this eigenfunction. For $n=1$ this is the Hecke zeta function of the elliptic modular form $F$; for $n=2$ the above product determines the Andrianov zeta function of the eigenfunction $F$ of genus 2. We shall restrict ourselves to the action on theta-series of the Hecke operators corresponding to the coefficients $\mathbf{q}_{1}^{n}(p), \mathbf{q}_{2^{n}-1}^{n}(p)$, $\mathbf{q}_{2^{n}}^{n}(p)$, and $\mathbf{q}_{2}^{2}(p)$. This will be sufficient for computation of the Euler product (3.23) corresponding to eigenfunctions of genus $n=1$ and 2. By using $\operatorname{An}(87)$ (3.3.81), 
(3.3.79), (3.3.80), and Exercise 3.3.38] and the above notation, these coefficients can be written in the form

$$
\begin{aligned}
\mathbf{q}_{1}^{n}(p) & =T^{n}(p), \\
\mathbf{q}_{2^{n}-1}(p) & =\left(p^{n(n+1) / 2}\langle p\rangle_{n}\right)^{2^{n-1}-1} T^{n}(p), \\
\mathbf{q}_{2^{n}}^{n}(p) & =\left(p^{n(n+1) / 2} T_{n}^{n}\left(p^{2}\right)\right)^{2^{n-1}}=\left(p^{n(n+1) / 2}\langle p\rangle_{n}\right)^{2^{n-1}}, \\
\mathbf{q}_{2}^{2}(p) & =p T_{1}^{2}\left(p^{2}\right)+p\left(p^{2}+1\right)\langle p\rangle_{2} .
\end{aligned}
$$

In particular, we have

$$
\begin{aligned}
\mathbf{Q}_{p}^{1}(t)= & \langle 1\rangle_{1}-T^{1}(p) t+p\langle p\rangle_{1} t^{2}, \\
\mathbf{Q}_{p}^{2}(t)= & \langle 1\rangle_{2}-T^{2}(p) t \\
& +\left(p T_{1}^{2}\left(p^{2}\right)+p\left(p^{2}+1\right)\langle p\rangle_{2}\right) t^{2}-p^{3}\langle p\rangle_{2} T^{2}(p) t^{3}+p^{6}\langle p\rangle_{2}^{2} t^{4} .
\end{aligned}
$$

Hence, it suffices to consider the action of the operators corresponding to the elements $T^{n}(p),\langle p\rangle_{n}$, and $T_{1}^{2}\left(p^{2}\right)$. By (3.14), (3.10), (3.20), and (1.14), we obtain

$$
\theta(Z ; P, Q) \mid T^{n}(p)=\delta_{n}^{m}(p) \theta(Z ; P \mid \tau(p), Q),
$$

where, unless $n \leq m / 2-1$ and $\chi_{Q}(p)=-1$, we have

$$
\begin{aligned}
\delta_{n}^{m}(p) & =p^{-n k+m / 2} \gamma_{n}^{m}(p)^{-1} \prod_{j=1}^{m / 2}\left(1+\chi_{Q}(p) p^{-j}\right) \\
& =\chi_{Q}(p)^{n} p^{-n\left(k+\frac{m}{2}\right)+\frac{n(n+1)}{2}} \times \begin{cases}\prod_{j=1}^{\frac{m}{2}-n}\left(1+\chi_{Q}(p) p^{j-1}\right)^{-1} & \text { if } n<m / 2 \text { and } \chi_{Q}(p) \neq-1, \\
1 & \text { if } n=m / 2, \\
\prod_{j=1}^{n-\frac{m}{2}}\left(1+\chi_{Q}(p) p^{-i}\right) & \text { if } n>m / 2 ;\end{cases}
\end{aligned}
$$

next, by (3.14), (3.12), and (3.21), we obtain

$$
\theta(Z ; P, Q) \mid\langle p\rangle_{n}=\chi_{Q}(p)^{n} p^{-n(m / 2+k)} \theta\left(Z ; P \mid[p]_{m}, Q\right) ;
$$

finally, if $m=4$, by (3.14), (3.11), (3.22), and (3.27), we get

$$
\begin{aligned}
& \theta(Z ; P, Q) \mid T_{1}^{2}\left(p^{2}\right) \\
&= \chi_{Q}(p) p^{-(3+4 k)} \theta\left(Z ; P \mid \tau\left[1, p, p, p^{2}\right], Q\right) \\
&+p^{2-4 k}\left(\alpha_{4}(p)-\chi_{Q}(p) p^{-6}\left(b_{4}(p)+p b_{3}(p)\right)\right) \theta\left(Z ; P \mid[p]_{4}, Q\right) \\
&= \chi_{Q}(p) p^{-(3+4 k)} \theta\left(Z ; P \mid \tau\left[1, p, p, p^{2}\right], Q\right) \\
&+p^{-4-4 k}\left(\chi_{Q}(p) p^{2}-1\right) \theta\left(Z ; P \mid[p]_{4}, Q\right) .
\end{aligned}
$$

We consider the action of Hecke operators on harmonic theta-series of genus $n=1$ in more detail. In this case,

$$
\begin{aligned}
T(p) & =T^{1}(p)=\sum_{M \in \Gamma_{0}(q) \backslash\left\{M \in \Sigma_{0}(q), \operatorname{det} M=p\right\}}\left(\Gamma_{0}(q) M\right) \\
& =\sum_{b=0}^{p-1}\left(\Gamma_{0}\left(\begin{array}{ll}
1 & b \\
0 & p
\end{array}\right)\right)+\left(\Gamma_{0}(q)\left(\begin{array}{ll}
p & 0 \\
0 & 1
\end{array}\right)\right),
\end{aligned}
$$

where $\Gamma_{0}(q)=\Gamma_{0}^{1}(q), \Sigma_{0}(q)=\Sigma_{0}^{1}(q)$, and

$$
\langle p\rangle=\langle p\rangle_{1}=\left(\left(\begin{array}{ll}
p & 0 \\
0 & p
\end{array}\right)\right)_{\Gamma_{0}(q)}=\left(\Gamma_{0}(q)\left(\begin{array}{ll}
p & 0 \\
0 & p
\end{array}\right)\right)
$$


for all prime numbers $p$ not dividing $q$. The definition shows that, for every modular form

$$
F=\sum_{a=0}^{\infty} f(a) e^{2 \pi i a z} \in \mathfrak{M}_{r}(q, \chi) \quad\left(z \in \mathbb{H}=\mathbb{H}_{1}\right)
$$

and every prime number $p$ not dividing $q$, we have

$$
\begin{aligned}
F \mid T(p) & =p^{-r} \sum_{b=0}^{p-1} F\left(\frac{z+b}{p}\right)+\chi(p) F(p z) \\
& =p^{1-r}\left(\sum_{a=0}^{\infty} f(a p) e^{2 \pi i a z}+\chi(p) p^{r-1} \sum_{a=0}^{\infty} f(a) e^{2 \pi i a p z}\right) .
\end{aligned}
$$

Let $Q$ be the matrix of a single-class positive definite quadratic form of level $q$ in an even number $m$ of variables, and let $P \in \mathcal{I}_{k}(Q)=\mathcal{I}_{k}^{1}(Q)$ be an invariant harmonic polynomial of weight (= degree) $k$ relative to $Q$. Let $p$ be a prime number not dividing $q$ and satisfying the condition $\chi_{Q}(p)=1$ if $m>2$. Then, by formula (3.26), the image of the theta-series $\theta(z ; P, Q) \in \mathfrak{M}_{k+m / 2}\left(q, \chi_{Q}\right)=\mathfrak{M}_{k+m / 2}^{1}\left(q, \chi_{Q}\right)$ under the Hecke operator $\mid T(p)$ can be written in the form

$$
\theta(z ; P, Q) \mid T(p)=\delta_{1}^{m}(p) \theta(z ; P \mid \tau(p), Q) .
$$

Since, by Proposition 1.6, the elements $\tau(p)$ with primes $p \nmid q$ commute with each other and, obviously, are invariant under the map (1.8), from Proposition 2.5 it follows that each of the spaces $\mathcal{I}_{k}(Q)$ has a basis of common eigenfunctions for all operators $\tau(p)$. If $P \in \mathcal{I}_{k}(Q)$ is such an eigenfunction with the eigenvalues $\lambda(\tau(p))$, then, by (3.30), the theta-series $\theta(z ; P, Q)$ is an eigenfunction for the Hecke operator $\mid T(p)$ with the eigenvalue

$$
\Lambda(T(p))=\delta_{1}^{m}(p) \lambda(\tau(p)) .
$$

So, we obtain the following statement.

Proposition 3.2. Let $Q$ be an even positive definite single-class matrix of even order $m=2 k$. Then each of the spaces

$$
\left\{\theta(z ; P, Q) \mid P \in \mathcal{I}_{k}(Q)\right\}
$$

has a basis of common eigenfunctions for all Hecke operators $T(p)$ with prime numbers $p$ not dividing the level of $Q$ and satisfying $\chi_{Q}(p)=1$ if $k>2$. The corresponding eigenvalues are of the form (3.31).

We consider an application of the above results to the case of theta-series with constant spherical functions. Clearly, for each positive definite matrix $Q$ the space $\mathcal{I}_{0}^{1}(Q)$ coincides with $\mathcal{P}_{0}^{1}(Q)$ and is generated by the constant function $P_{0}(X)$ identically equal to 1 . The function $P_{0}$ is obviously an eigenfunction for all Hecke operators $\mid \tau(d)$ of the form (2.13) corresponding to the elements $\tau(d)$ of the form (1.14), with the eigenvalues given by

$$
\lambda(\tau(d))=\#(A(Q, d)) / \#(E(Q)) \quad(\operatorname{gcd}(d, \operatorname{det} Q)=1) .
$$

The theta-series of genus $n=1$ with spherical polynomial $P_{0}$ is the ordinary theta-series of the quadratic form q with matrix $Q$,

$$
\theta(z ; Q)=\theta\left(z ; P_{0}, Q\right)=\sum_{N \in \mathbb{Z}^{m}} e^{2 \pi i \mathbf{q}(N) z}=\sum_{a=0}^{\infty} r(a, Q) e^{2 \pi i a z},
$$

whose $a$ th Fourier coefficient $r(a, Q)$ is equal to the number of solutions of the equation $\mathbf{q}(X)=a$ in integral vectors $X=N \in \mathbb{Z}^{m}$. By Proposition 3.2, the theta-series (3.33) is an eigenfunction for the Hecke operator $\mid T(p)$,

$$
\theta(z ; Q) \mid T(p)=\Lambda(T(p)) \theta(z ; Q) .
$$


Comparing the constant terms of the series on both sides of (3.29), we obtain

$$
\Lambda(T(p))=p^{1-m / 2}\left(1+\chi_{Q}(p) p^{m / 2-1}\right) .
$$

By (3.32) and (3.26), this leads to the formula

$$
\frac{\#(A(Q, p))}{\#(E(Q))}=\lambda(\tau(p))=\lambda(T(p)) \delta_{1}^{m}(p)^{-1}= \begin{cases}1+\chi_{q}(p) & \text { if } m=2 \\ \prod_{i=0}^{m / 2-1}\left(1+p^{i}\right) & \text { if } m>2\end{cases}
$$

Now, we consider the relationship between the zeta functions (0.7) corresponding to eigenfunctions of automorph class rings of binary and quaternary quadratic forms on spaces of harmonic forms and the zeta functions (3.23) of the theta-series weighted by these harmonic forms.

Let $Q$ be the matrix of a single-class positive definite quadratic form in two variables, and let $P \in \mathcal{I}_{k}^{1}(Q)$ be an eigenfunction for all Hecke operators $\mid \tau(p)$ with prime numbers $p$ not dividing the level $q$ of $Q$. By our assumption and (2.4), for these primes we have

$$
P \mid \tau(p)=\lambda(\tau(p)) P \quad \text { and } \quad P \mid[p]=P(p X)=p^{k} P=\lambda([p]) P .
$$

Consequently, the $p$-factor of the zeta function (0.7) of $P$ looks like this:

$$
Z_{p}(t, \lambda)=\left(1-\lambda(\tau(p)) p^{-s}+\chi_{Q}(p) \lambda([p]) p^{-2 s}\right)^{-1} .
$$

On the other hand, by (3.26) and (3.27), the theta-series $\theta(z ; P, Q) \in \mathfrak{M}_{1+k}^{1}\left(q, \chi_{Q}\right)$ is an eigenfunction of the Hecke operators $|T(p)=| T^{1}(p)$ and $|\langle p\rangle=|\langle p\rangle_{1}$ with the eigenvalues

$$
\begin{aligned}
\Lambda(T(p)) & =\delta_{1}^{2}(p) \lambda(\tau(p))=\chi_{Q}(p) p^{-k} \lambda(\tau(p)), \\
\Lambda(\langle p\rangle) & =\chi_{Q}(p) p^{-(1+k)}=\chi_{Q}(p) p^{-(1+2 k)} \lambda([p]),
\end{aligned}
$$

respectively. It follows that, for the $p$-factor of the zeta function $(3.23)$ with character $\psi=\chi_{Q}$ of the eigenfunction $\theta(z ; P, Q)$, we obtain the identity

$$
\begin{gathered}
\mathbf{Q}_{p}^{1}\left(\chi_{Q}(p) p^{k-s}, \Lambda\right)^{-1}=\left(1-\Lambda(T(p)) \chi_{Q}(p) p^{k-s}+\Lambda(\langle p\rangle) p\left(\chi_{Q}(p) p^{k-s}\right)^{2}\right)^{-1} \\
=\left(1-\lambda(\tau(p)) p^{-s}+\chi_{Q}(p) \lambda([p]) p^{-2 s}\right)^{-1}=Z_{p}(t, P) .
\end{gathered}
$$

These identities for the local zeta functions for all primes $p$ not dividing the level of $Q$ imply equality of the Euler products (0.7) and (3.23).

Theorem 3.3. Let $Q$ be the matrix of a single-class positive definite binary quadratic form, let $q$ be the level of $Q$, and let $\chi_{Q}$ be the corresponding Dirichlet character modulo $q$. If $P \in \mathcal{I}_{k}^{1}(Q)$ is a harmonic eigenform for all Hecke operators $\mid \tau(p)$ with prime numbers $p \nmid q$, then the theta-series of $Q$ with harmonic form $P$,

$$
F=\theta(z ; P, Q) \in \mathfrak{M}_{1+k}^{1}\left(q, \chi_{Q}\right),
$$

is an eigenfunction for all Hecke operators $|T(p)=| T^{1}(p)$ with primes $p$ as above, and the zeta functions (0.7) and (3.23) of these eigenfunctions are related by the identity

$$
Z^{*}(s, P)=Z_{F}\left(s-k, \chi_{Q}\right) .
$$

Finally, we prove that similar relations are valid also in the case of quadratic forms in $m=4$ variables, for harmonic forms of genus $n=2$ and the corresponding theta-series.

Theorem 3.4. Let $Q$ be the matrix of a single-class positive definite quaternary quadratic form, let $q$ be the level of $Q$, and let $\chi_{Q}$ be the corresponding Dirichlet character modulo $q$. If $P \in \mathcal{I}_{k}^{2}(Q)$ is a harmonic eigenform for all Hecke operators $\mid \tau(p)$ and $\mid \tau\left[1, p, p, p^{2}\right]$ with prime numbers $p \nmid q$, then the theta-series of $Q$ with harmonic form $P$,

$$
F=\theta(Z ; P, Q) \in \mathfrak{M}_{2+k}^{2}\left(q, \chi_{Q}\right)
$$


is an eigenfunction for all Hecke operators $\mid T^{1}(p)$ and $T_{1}^{2}\left(p^{2}\right)$ with primes $p$ as above, and the zeta functions (0.7) and (3.23) of these eigenfunctions are related by the identity

$$
Z^{*}(s, P)=Z_{F}\left(s-2 k-1, \chi_{0}\right),
$$

where $\chi_{0}$ is the unit character modulo $q$.

Proof. By assumption, for each prime number $p$ with $p \nmid q$ we have

$$
P|\tau(p)=\lambda(\tau(p)) P, \quad P| \tau\left[1, p, p, p^{2}\right]=\lambda\left(\tau\left[1, p, p, p^{2}\right]\right) P .
$$

By (2.4),

$$
P \mid[p]_{4}=P(p X)=p^{2 k} P=\lambda\left([p]_{4}\right) P .
$$

Consequently, by (1.28) and (1.25), the $p$-factor of the orthogonal zeta function (0.7) of the eigenform $P$ looks like this:

$$
\begin{aligned}
Z_{p}(t, \lambda)=\left(1-\lambda(\tau(p)) p^{-s}+\left(\chi_{Q}(p) \lambda\left(\tau\left[1, p, p, p^{2}\right]\right)+\left(1+\chi_{Q}(p)\right) p \lambda\left([p]_{2}\right)\right) p^{-2 s}\right. \\
\left.-p \lambda\left([p]_{2}\right) \lambda(\tau(p)) p^{-3 s}+p^{2} \lambda\left([p]_{2}\right)^{2} p^{-4 s}\right)^{-1} .
\end{aligned}
$$

On the other hand, using (3.26), (3.27), and (3.28), we obtain

$$
\begin{aligned}
& \theta(Z ; P, Q) \mid T^{2}(p)=\delta_{2}^{4}(p) \theta(Z ; P \mid \tau(p), Q)=p^{-2 k-1} \lambda(\tau(p)) \theta(Z ; P, Q), \\
& \theta(Z ; P, Q) \mid\langle p\rangle_{2}=\chi_{Q}(p)^{2} p^{-2(k+2)} \theta\left(Z ; P \mid[p]_{4}, Q\right)=p^{-4 k-4} \lambda\left([p]_{4}\right) \theta(Z ; P, Q),
\end{aligned}
$$

and

$$
\begin{aligned}
\theta(Z ; & P, Q) \mid T_{1}^{2}\left(p^{2}\right) \\
& =\chi_{Q}(p) p^{-(4 k+3)} \theta\left(Z ; P \mid \tau\left[1, p, p, p^{2}\right], Q\right)+p^{-4 k-4}\left(\chi_{Q}(p) p^{2}-1\right) \theta\left(Z ; P \mid[p]_{4}, Q\right) \\
& =\left(\chi_{Q}(p) p^{-(4 k+3)} \lambda\left(\tau\left[1, p, p, p^{2}\right]\right)+p^{-4 k-4}\left(\chi_{Q}(p) p^{2}-1\right) \lambda\left([p]_{4}\right)\right) \theta(Z ; P, Q) .
\end{aligned}
$$

Hence, by (3.25), we conclude that the $p$-factor of the zeta function (3.23) with $s-2 k-1$ in place of $s$ and $\psi=\chi_{0}$, corresponding to the eigenfunction $F=\theta(Z ; P, Q)$ of the Hecke operators $\mid T$ in the ring $\mathcal{H}_{0}^{2}(q)$, is equal to

$$
\begin{aligned}
Q\left(p^{2 k+1-s}, \Lambda\right)=\left(1-\Lambda\left(T^{2}(p)\right) t+\right. & \Lambda \\
& \left.p T_{1}^{2}\left(p^{2}\right)+p\left(p^{2}+1\right)\langle p\rangle_{2}\right) t^{2} \\
& \left.-\Lambda\left(p^{3}\langle p\rangle_{2} T^{2}(p)\right) t^{3}+p^{6} \Lambda\left(\langle p\rangle_{2}^{2}\right) t^{4}\right)^{-1}
\end{aligned}
$$

where the $\Lambda(T)$ are the corresponding eigenvalues and $t=p^{2 k+1-s}$. Thus, we have

$$
\begin{aligned}
\mathbf{Q}\left(p^{2 k+1-s}, \Lambda\right)= & \left(1-p^{-2 k-1} \lambda(\tau(p)) t\right. \\
& +\left(p \chi_{Q}(p) p^{-(4 k+3)} \lambda\left(\tau\left[1, p, p, p^{2}\right]\right)+p^{-4 k-4} p\left(\chi_{Q}(p) p^{2}-1\right) \lambda\left([p]_{4}\right)\right. \\
& \left.+p\left(p^{2}+1\right) p^{-4 k-4} \lambda\left([p]_{4}\right)\right) t^{2} \\
& \left.\quad+p^{3} p^{-4 k-4} \lambda\left([p]_{4}\right) p^{-2 k-1} \lambda(\tau(p)) t^{3}+p^{6} p^{-8 k-8} \lambda\left([p]_{4}\right)^{2} t^{4}\right)^{-1} \\
= & \left(1-\lambda(\tau(p)) p^{-s}+\left(\chi_{Q}(p) \lambda\left(\tau\left[1, p, p, p^{2}\right]\right)+\left(1+\chi_{Q}(p)\right) p \lambda\left([p]_{4}\right)\right) p^{-2 s}\right. \\
= & \left.-p \lambda\left([p]_{4}\right) \lambda(\tau(p)) p^{-3 s}+p^{2} \lambda\left([p]_{4}\right)^{2} p^{-4 s}\right)^{-1} \\
= & Z_{p}(t, \lambda) .
\end{aligned}
$$

These identities for the local zeta functions for all primes $p$ not dividing the level of $Q$ imply that the Euler products (0.7) and (3.23) are equal. 


\section{$\S 4$. EXAmples}

1. Let $Q=\left(\begin{array}{ll}2 & 0 \\ 0 & 2\end{array}\right)$ be the matrix of the sum of two squares

$$
\mathbf{q}(X)=\frac{1}{2}{ }^{t} X Q X=x_{1}^{2}+x_{2}^{2} \quad\left(X=\left(\begin{array}{l}
x_{1} \\
x_{2}
\end{array}\right)\right) .
$$

An integral matrix $D=\left(\begin{array}{ll}a & b \\ c & d\end{array}\right)$ is an automorph of $\mathbf{q}$ with multiplier $\mu$ if and only if $a^{2}+c^{2}=b^{2}+d^{2}=\mu$ and $a b+c d=0$. Hence, if $\mu=0$, then $D=0$, but if $\mu \neq 0$, then $\mu>0,(b, d)=\lambda(-c, a)$, and $\lambda^{2}=1$, so that $D=\left(\begin{array}{cc}a & -c \\ c & a\end{array}\right)$ or $D=\left(\begin{array}{cc}a & c \\ c & -a\end{array}\right)$. We conclude that the set of proper automorphs of $\mathbf{q}$ has the form

$$
\mathbf{A}=A(\mathbf{q})=\bigcup_{\mu=0}^{\infty} A(\mathbf{q}, \mu) \quad \text { with } A(\mathbf{q}, \mu)=\left\{D=\left(\begin{array}{cc}
a & -c \\
c & a
\end{array}\right) \mid a, c \in \mathbb{Z}, a^{2}+c^{2}=\mu\right\},
$$

and the group of proper units is

$$
\mathbf{E}=E(\mathbf{q})=\left\{U= \pm\left(\begin{array}{ll}
1 & 0 \\
0 & 1
\end{array}\right), \pm\left(\begin{array}{cc}
0 & -1 \\
1 & 0
\end{array}\right)\right\}
$$

It follows that the set of matrices of proper automorphs of $\mathbf{q}$ is a ring with respect to the standard matrix addition and multiplication, and it can easily be checked that the mapping

$$
\alpha: D=\left(\begin{array}{cc}
a & -c \\
c & a
\end{array}\right) \mapsto \alpha(D)=a-\sqrt{-1} c
$$

is an isomorphism between the ring $\mathbf{A}$ and the ring $\mathcal{O}=\mathbb{Z}[\sqrt{-1}]$ of Gaussian integers; moreover, the group $\mathbf{E}$ is mapped onto the group of units of $\mathcal{O}$. In particular, each double coset $\mathbf{E} D \mathbf{E} \subset \mathbf{A}$ coincides with the left coset $\mathbf{E} D$, and the principal ideals $\alpha(D) \mathcal{O}$ are in one-to-one correspondence with the double or left cosets. This correspondence, which will still be denoted by $\alpha$, so that $\alpha(\mathbf{E} D \mathbf{E})=\alpha(D) \mathcal{O}$, is clearly compatible with multiplication of double cosets in the automorph class ring $\mathcal{H}=\mathcal{H}(\mathbf{q})$ and the usual multiplication of ideals in $\mathcal{O}$, and

$$
N(\alpha(D) \mathcal{O})=N(\alpha(D))=\mu(D),
$$

where $N$ stands for the norm. Hence, the linear extension of this correspondence to the entire $\mathcal{H}$ is an isomorphism of the ring $\mathcal{H}$ onto the semigroup ring over $\mathbb{Z}$ of the semigroup of nonzero ideals of the ring $\mathcal{O}$ (note that all ideals of $\mathcal{O}$ are principal). This allows us to rewrite the formal Dirichlet series (0.5) in terms of ideals:

$$
\sum_{\mu=1}^{\infty} \frac{\tau(\mu)}{\mu^{s}} \Longrightarrow \sum_{\mu=1}^{\infty} \frac{\alpha(\tau(\mu))}{\mu^{s}}=\sum_{\mathfrak{a}} \frac{\mathfrak{a}}{N(\mathfrak{a})^{s}}
$$

where $\mathfrak{a}$ ranges over all nonzero ideals of $\mathcal{O}$, and use the multiplicative theory of ideals for Euler factorization of the series. We get the formal Euler factorization of the last-written series in the form

$$
\prod_{\mathfrak{p}} \sum_{\delta=0}^{\infty} \frac{\mathfrak{p}^{\delta}}{N(\mathfrak{p})^{\delta s}}=\prod_{\mathfrak{p}}\left((1)-\frac{\mathfrak{p}}{N(\mathfrak{p})^{s}}\right)^{-1}=\prod_{p} \prod_{\mathfrak{p} \mid p}\left((1)-\frac{\mathfrak{p}}{N(\mathfrak{p})^{s}}\right)^{-1},
$$

where $\mathfrak{p}$ runs through all prime ideals of $\mathcal{O},(1)=\mathcal{O}$ is the unit ideal, $p$ runs through all rational prime numbers, and in the last product $\mathfrak{p}$ ranges over all prime ideals dividing the ideal $(p)=p \mathcal{O}$. We recall the well-known laws of factorization of prime numbers into prime ideals of the ring $\mathcal{O}$. If $p=2$, there is only one prime ideal $\mathfrak{p}=(1+\sqrt{-1})$ dividing $(p),(p)=\mathfrak{p}^{2}, N(\mathfrak{p})=p$, and $\mathfrak{p}=\alpha(\tau(p))$. If $p$ is odd and $\chi_{Q}(p)=(-1)^{\frac{p-1}{2}}=1$, then there are two conjugate prime ideals $\mathfrak{p}=(a+\sqrt{-1} c)$ and $\overline{\mathfrak{p}}=(a-\sqrt{-1} c)$ dividing 
$(p)$, where $a^{2}+c^{2}=p,(p)=\mathfrak{p} \overline{\mathfrak{p}}, N(\mathfrak{p})=N(\overline{\mathfrak{p}})=p, \mathfrak{p}+\overline{\mathfrak{p}}=\alpha(\tau(p))$, and $\left.\mathfrak{p} \overline{\mathfrak{p}}=\alpha([p])\right)$. Finally, if $p$ is odd and $\chi_{Q}(p)=(-1)^{\frac{p-1}{2}}=-1$, then the ideal $(p)=\mathfrak{p}$ is prime, $N(\mathfrak{p})=p^{2}$, $\alpha(\tau(p))=0$, and $\mathfrak{p}=\alpha([p])$. Therefore, the Euler product expansion can be rewritten in terms of automorphs as

$$
\sum_{\mu=1}^{\infty} \frac{\tau(\mu)}{\mu^{s}}=\left([1]-\frac{\tau(2)}{2^{s}}\right)^{-1} \prod_{p \text { odd primes }}\left([1]-\frac{\tau(p)}{p^{s}}+\frac{\chi_{Q}(p)[p]}{p^{2 s}}\right)^{-1} .
$$

Now we turn to the representation of the ring $\mathcal{H}=\mathcal{H}(\mathbf{q})$ on the space $\mathcal{P}_{k}=\mathcal{P}_{k}^{1}(Q)$ of harmonic forms of genus 1 and weight $k$ relative to $Q$. Using Proposition 2.3, we see that the space $\mathcal{P}_{k}$ for $k>1$ is spanned over $\mathbb{C}$ by the forms

$$
P_{k}(X)=\left(x_{1}-\sqrt{-1} x_{2}\right)^{k} \text { and } \bar{P}_{k}(X)=\left(x_{1}+\sqrt{-1} x_{2}\right)^{k} \quad\left(X=\left(\begin{array}{l}
x_{1} \\
x_{2}
\end{array}\right)\right) .
$$

It is easy to check that the subspace $\mathcal{I}_{k}=\mathcal{I}_{k}^{1}(Q)$ of $\mathbf{E}$-invariant forms contain a nonzero form if and only if $k$ is divisible by 4 , and in this case $\mathcal{I}_{k}=\mathcal{P}_{k}$. The image of a form $P \in \mathcal{P}_{k}$, where $k$ is divisible by 4 , under the action (2.13) of the Hecke operator $\mid \tau(D)$ corresponding to a basic element (1.6) of the ring $\mathcal{H}$ is equal to

$$
P \mid \tau(D)=\sum_{D^{\prime} \in \mathbf{E} \backslash \mathbf{E} D \mathbf{E}} P\left(D^{\prime} X\right)=P(D X),
$$

because $\mathbf{E} D \mathbf{E}=\mathbf{E} D$ and $\mathcal{P}_{k}=\mathcal{I}_{k}$. If $P=P_{k}$ and $D=\left(\begin{array}{cc}a & -c \\ c & a\end{array}\right)$, then, by (4.4), we have

$$
\begin{aligned}
P_{k} \mid \tau(D) & =P_{k}(D X)=\left(a x_{1}-c x_{2}-\sqrt{-1}\left(c x_{1}+a x_{2}\right)\right)^{k} \\
& =(a-\sqrt{-1} c)^{k}\left(x_{1}-\sqrt{-1} x_{2}\right)^{k}=\alpha(D)^{k} P_{k},
\end{aligned}
$$

where $\alpha$ is the mapping (4.1). Similarly,

$$
\bar{P}_{k} \mid \tau(D)=\bar{P}_{k}(D X)=(a+\sqrt{-1} c)^{k}\left(x_{1}+\sqrt{-1} x_{2}\right)^{k}=\bar{\alpha}(D)^{k} \bar{P}_{k} .
$$

Thus, the polynomials (4.3) present a basis of eigenfunctions for all Hecke operators on $\mathcal{P}_{k}$. In particular, it is a basis of eigenfunctions of the Hecke operators $\mid \tau(p)$ and $[p]$ for all prime numbers $p$. By the decomposition laws cited above and formulas (4.5), (4.6), and (2.4), we obtain the formulas

$$
P \mid \tau(p)=\lambda_{k}(p) P \quad \text { and } \quad P \mid[p]=p^{k} P,
$$

where

$$
\lambda_{k}(p)= \begin{cases}(1+\sqrt{-1})^{k}=(1-\sqrt{-1})^{k} & \text { if } p=2, \\ (a+\sqrt{-1} c)^{k}+(a-\sqrt{-1} c)^{k} & \text { if }(-1)^{\frac{p-1}{2}}=1 \text { and } a^{2}+c^{2}=p, \\ 0 & \text { if }(-1)^{\frac{p-1}{2}}=-1 ;\end{cases}
$$

formulas (4.7) are valid for $P=P_{k}, \bar{P}_{k}$ and hence for every $P \in \mathcal{P}_{k}$. It follows that, for every positive integer $\mu$ and $P \in \mathcal{P}_{k}$, we have

$$
P \mid \tau(\mu)=\lambda_{k}(\mu) P,
$$

where $\lambda_{k}$ is the totally multiplicative function on $\mathbb{N}$ given on prime numbers by (4.8). Thus, we obtain the Euler product presentation of the global orthogonal zeta function of the quadratic form $\mathbf{q}$ with the character $\lambda(\tau(\mu))=\lambda_{k}(\mu)$ :

$$
\begin{aligned}
Z(s, P) & =\sum_{\mu=1}^{\infty} \frac{\lambda_{k}(\mu)}{\mu^{s}} \\
& =\left(1-\frac{\lambda_{k}(2)}{2^{s}}\right)^{-1} \prod_{p \text { odd primes }}\left(1-\frac{\lambda_{k}(p)}{p^{s}}+\frac{\chi_{Q}(p) p^{k}}{p^{2 s}}\right)^{-1} .
\end{aligned}
$$


Returning to the ring $\mathcal{O}$ of Gaussian integers, we can interpret this identity in terms of ideals of $\mathcal{O}$ rather than automorphs of $\mathbf{q}$. For $l$ divisible by 4 , let $\Psi_{l}$ denote the Hecke character of the semigroup of nonzero ideals of $\mathcal{O}$ given by

$$
\Psi_{l}(\mathfrak{a})=\Psi_{l}((\alpha))=\left(\frac{\alpha}{|\alpha|}\right)^{l}
$$

Then, since

$$
\lambda_{k}(\mathfrak{a})=\lambda_{k}((\alpha))=\alpha^{k}=(\alpha \bar{\alpha})^{k / 2}\left(\frac{\alpha}{|\alpha|}\right)^{k}=N(\mathfrak{a})^{k / 2} \Psi_{k}(\mathfrak{a}) \quad(k \equiv 0(\bmod 4)),
$$

it is not hard to show that the Euler product above can be written in the form

$$
\prod_{\mathfrak{p}}\left(1-\frac{\Psi_{k}(\mathfrak{p})}{N(\mathfrak{p})^{s-k / 2}}\right)^{-1}
$$

where $\mathfrak{p}$ runs through all prime ideals of $\mathcal{O}$. Thus, finally, we arrive at the identity

$$
Z(s, P)=\zeta_{\mathcal{O}}\left(s-k / 2, \Psi_{k}\right),
$$

where the function

$$
\zeta_{\mathcal{O}}\left(s, \Psi_{k}\right)=\sum_{\mathfrak{a}} \frac{\Psi_{k}(\mathfrak{a})}{N(\mathfrak{a})^{s}}=\prod_{\mathfrak{p}}\left(1-\frac{\Psi_{k}(\mathfrak{p})}{N(\mathfrak{p})^{s}}\right)^{-1},
$$

with $\mathfrak{a}$ running through all nonzero ideals of $\mathcal{O}$, is the Hecke zeta function of the ring $\mathcal{O}$ with the character $\Psi_{k}$. It follows that the Dirichlet series $Z\left(s, \lambda_{k}, \mathbf{q}\right)$ converges absolutely and uniformly in each right half-plane $\operatorname{Re} s \geq 1+k / 2+\epsilon$ with $\epsilon>0$. In every domain of absolute convergence, the function $Z\left(s, \lambda_{k}, \mathbf{q}\right)$ can be presented in terms of the Mellin integral of the theta-series $\theta\left(z ; P_{k}, Q\right)$,

$$
r(2, P, Q) Z(s, P)=\frac{(2 \pi)^{s}}{\Gamma(s)} \int_{0}^{\infty}(\theta(\sqrt{-1} t ; P, Q)-P(0)) t^{s-1} d t,
$$

where $r(2, P, Q)$ is the coefficient of $e^{2 \pi i z}$ in the expansion (3.2) of the theta-series, and $\Gamma(s)$ is the gamma function. This representation makes it possible to prove that the zeta function admits meromorphic analytic continuation to the entire $s$-plane and satisfies a functional equation. Alternatively, the analytic properties of the zeta function $\zeta_{\mathcal{O}}\left(s, \Psi_{k}\right)$ can be investigated in the spirit of J. Tate with the help of Fourier analysis on the adele space of the field $\mathbb{Q}(\sqrt{-1})$.

2. Now we consider another important single-class quadratic form: let

$$
\mathbf{q}(X)=\frac{1}{2}{ }^{t} X Q X=x_{1}^{2}-x_{1} x_{2}+x_{2}^{2}=\left(x_{1}-\rho x_{2}\right)\left(x_{1}-\bar{\rho} x_{2}\right), \quad \text { where } \rho=\frac{1+\sqrt{-3}}{2},
$$

with the matrix $Q=\left(\begin{array}{cc}2 & -1 \\ -1 & 2\end{array}\right)$. An integral matrix $D=\left(\begin{array}{ll}a & b \\ c & d\end{array}\right)$ is a proper automorph of q with multiplier $\mu$ if and only if $\operatorname{det} D=a d-b c>0$ and

$$
\begin{aligned}
\mathbf{q}(D X) & =\left(a x_{1}+b x_{2}-\rho\left(c x_{1}+d x_{2}\right)\right)\left(a x_{1}+b x_{2}-\bar{\rho}\left(c x_{1}+d x_{2}\right)\right) \\
& =(a-\rho c)(a-\bar{\rho} c)\left(x_{1}-\frac{-b+\rho d}{a-\rho c} x_{2}\right)\left(x_{1}-\frac{-b+\bar{\rho} d}{a-\bar{\rho} c} x_{2}\right) \\
& =\mu\left(x_{1}-\rho x_{2}\right)\left(x_{1}-\bar{\rho} x_{2}\right),
\end{aligned}
$$

i.e., $(a-\rho c)(a-\bar{\rho} c)=a^{2}-a c+c^{2}=\mu$ and $(-b+\rho d)(a-\rho c)^{-1}=\rho$, because both $\rho$ and $(-b+\rho d)(a-\rho c)^{-1}$ with $a d-b c>0$ belong to the upper complex half-plane. The last relation means that

$$
-b+\rho d=\rho(a-\rho c)=\rho a-(\rho-1) c=c+\rho(a-c), \text { i.e., } b=-c, d=a-c .
$$


We conclude that the set of proper automorphs of $\mathbf{q}$ has the form

$$
\mathbf{A}=A(\mathbf{q})=\bigcup_{\mu=0}^{\infty} A(\mathbf{q}, \mu)
$$

with

$$
A(\mathbf{q}, \mu)=\left\{D=\left(\begin{array}{cc}
a & -c \\
c & a-c
\end{array}\right) \mid a, c \in \mathbb{Z}, a^{2}-a c+c^{2}=\mu\right\},
$$

and the group of proper units is

$$
\mathbf{E}=E(\mathbf{q})=\left\{U= \pm\left(\begin{array}{ll}
1 & 0 \\
0 & 1
\end{array}\right), \pm\left(\begin{array}{cc}
0 & -1 \\
1 & -1
\end{array}\right), \pm\left(\begin{array}{cc}
1 & 1 \\
-1 & 0
\end{array}\right)\right\} .
$$

It follows that the matrices of the proper automorphs of $\mathbf{q}$ form a ring with respect to the standard matrix addition and multiplication, and it is easily seen that the mapping

$$
\beta: D=\left(\begin{array}{cc}
a & -c \\
c & a-c
\end{array}\right) \mapsto \beta(D)=a-\rho c
$$

is an isomorphism of the ring $\mathbf{A}$ into the ring $\mathcal{O}=\mathbb{Z}[\rho]$ of integers of the quadratic extension $K=\mathbb{Q}(\rho)$ of the rational number field; moreover, the group $\mathbf{E}$ is mapped onto the group of units of $\mathcal{O}$. In particular, each double coset $\mathbf{E} D \mathbf{E} \subset \mathbf{A}$ coincides with the left coset $\mathbf{E} D$, and the principal ideals $\beta(D) \mathcal{O}$ are in one-to-one correspondence with the double or left cosets. This correspondence, which will still be denoted by $\beta$, so that $\beta(\mathbf{E} D \mathbf{E})=\beta(D) \mathcal{O}$, is clearly compatible with multiplication of double cosets in the automorph class ring $\mathcal{H}=\mathcal{H}(\mathbf{q})$ and the usual multiplication of ideals in $\mathcal{O}$, and

$$
N(\beta(D) \mathcal{O})=N(\beta(D))=\mu(D),
$$

where $N$ stands for the norm in the field $K$. Hence, the linear extension of this correspondence to the entire ring $\mathcal{H}$ is an isomorphism of $\mathcal{H}$ onto the semigroup ring over $\mathbb{Z}$ of the semigroup of nonzero ideals of the ring $\mathcal{O}$ (note that all ideals of $\mathcal{O}$ are principal). This allows us to rewrite the formal Dirichlet series (0.5) in terms of ideals:

$$
\sum_{\mu=1}^{\infty} \frac{\tau(\mu)}{\mu^{s}} \Longrightarrow \sum_{\mu=1}^{\infty} \frac{\alpha(\tau(\mu))}{\mu^{s}}=\sum_{\mathfrak{a}} \frac{\mathfrak{a}}{N(\mathfrak{a})^{s}},
$$

where $\mathfrak{a}$ ranges over all nonzero ideals of $\mathcal{O}$, and we can use the multiplicative theory of ideals for Euler factorization of series. We get the formal Euler product presentation of the last-written series in the form

$$
\prod_{\mathfrak{p}} \sum_{\delta=0}^{\infty} \frac{\mathfrak{p}^{\delta}}{N(\mathfrak{p})^{\delta s}}=\prod_{\mathfrak{p}}\left((1)-\frac{\mathfrak{p}}{N(\mathfrak{p})^{s}}\right)^{-1}=\prod_{p} \prod_{\mathfrak{p} \mid p}\left((1)-\frac{\mathfrak{p}}{N(\mathfrak{p})^{s}}\right)^{-1}
$$

where $\mathfrak{p}$ runs through all prime ideals of $\mathcal{O},(1)=\mathcal{O}$ is the unit ideal, $p$ runs through all rational prime numbers, and $\mathfrak{p}$ in the rightmost product ranges over all prime ideals dividing the ideal $(p)=p \mathcal{O}$. We use the well-known laws of factorization of prime numbers into prime ideals of the ring $\mathcal{O}=\mathbb{Z}[\rho]$. If $p=3$, there is only one prime ideal $\mathfrak{p}=(1-\rho)=(1-\bar{\rho})$ dividing $(p),(p)=\mathfrak{p}^{2}, N(\mathfrak{p})=p$, and $\mathfrak{p}=\beta(\tau(p))$. If $p \neq 3$ and $\chi_{Q}(p)=1$, then there are two conjugate prime ideals $\mathfrak{p}=(a+\rho c)$ and $\overline{\mathfrak{p}}=(a+\bar{\rho} c)$ dividing $(p)$, where $a^{2}-a c+c^{2}=p,(p)=\mathfrak{p} \overline{\mathfrak{p}}, N(\mathfrak{p})=N(\overline{\mathfrak{p}})=p, \mathfrak{p}+\overline{\mathfrak{p}}=\alpha(\tau(p))$, and $\mathfrak{p} \overline{\mathfrak{p}}=\alpha([p]))$. Finally, if $p$ is odd and $\chi_{Q}(p)=-1$, then the ideal $(p)=\mathfrak{p}$ is prime, $N(\mathfrak{p})=p^{2}, \alpha(\tau(p))=0$, and $\mathfrak{p}=\alpha([p])$. Therefore, the Euler product expansion can be rewritten in terms of automorphs as

$$
\sum_{\mu=1}^{\infty} \frac{\tau(\mu)}{\mu^{s}}=\left([1]-\frac{\tau(3)}{3^{s}}\right)^{-1} \prod_{p \text { primes, } p \neq 3}\left([1]-\frac{\tau(p)}{p^{s}}+\frac{\chi_{Q}(p)[p]}{p^{2 s}}\right)^{-1} .
$$


We turn to the representation of the ring $\mathcal{H}=\mathcal{H}(Q)$ on the space $\mathcal{P}_{k}=\mathcal{P}_{k}^{1}(Q)$ of harmonic forms of genus 1 and weight $k$ relative to $Q$. Using Proposition 2.3, we see that the space $\mathcal{P}_{k}$ for $k>1$ is spanned over $\mathbb{C}$ by the forms

$$
P_{k}(X)=\left(x_{1}-\rho x_{2}\right)^{k} \text { and } \bar{P}_{k}(X)=\left(x_{1}-\bar{\rho} x_{2}\right)^{k} \quad\left(X=\left(\begin{array}{l}
x_{1} \\
x_{2}
\end{array}\right)\right) .
$$

It is easy to check that the subspace $\mathcal{I}_{k}=\mathcal{I}_{k}^{1}(Q)$ of $\mathbf{E}$-invariant forms of $\mathcal{P}_{k}$ contains a nonzero form if and only if $k$ is divisible by 6 , and in this case $\mathcal{I}_{k}=\mathcal{P}_{k}$. The image of a form $P \in \mathcal{P}_{k}$, where $k$ is divisible by 6 , under the action (2.13) of the Hecke operator $\mid \tau(D)$ corresponding to a basic element (1.6) of the ring $\mathcal{H}$ is equal to

$$
P \mid \tau(D)=\sum_{D^{\prime} \in \mathbf{E} \backslash \mathbf{E} D \mathbf{E}} P\left(D^{\prime} X\right)=P(D X),
$$

because $\mathbf{E} D \mathbf{E}=\mathbf{E} D$ and $\mathcal{P}_{k}=\mathcal{I}_{k}$. If $P=P_{k}$ and $D=\left(\begin{array}{cc}a & -c \\ c & a-c\end{array}\right)$, then, by (4.14), we have

$$
\begin{aligned}
P_{k} \mid \tau(D) & =P_{k}(D X)=\left(a x_{1}-c x_{2}+\rho\left(c x_{1}+(a-c) x_{2}\right)\right)^{k} \\
& =(a-\rho c)^{k}\left(x_{1}+\rho x_{2}\right)^{k}=\beta(D)^{k} P_{k},
\end{aligned}
$$

where $\beta$ is the mapping (4.11). Similarly,

$$
\bar{P}_{k} \mid \tau(D)=\bar{P}_{k}(D X)=(a-\bar{\rho} c)^{k}\left(x_{1}-\bar{\rho} x_{2}\right)^{k}=\bar{\beta}(D)^{k} \bar{P}_{k} .
$$

Thus, the polynomials (4.13) present a basis of eigenfunctions for all Hecke operators on $\mathcal{P}_{k}$. In particular, it is a basis of eigenfunctions of the Hecke operators $\mid \tau(p)$ and $[p]$ for all prime numbers $p$. By the decomposition laws cited above and formulas (4.15), (4.16), and (2.4), we obtain the formulas

$$
P \mid \tau(p)=\lambda_{k}(p) P \quad \text { and } \quad P \mid[p]=p^{k} P,
$$

where

$$
\lambda_{k}(p)= \begin{cases}(1-\rho)^{k}=(1-\bar{\rho})^{k} & \text { if } p=3 \\ (a-\rho c)^{k}+(a-\bar{\rho} c)^{k} & \text { if } \chi_{Q}(p)=1 \text { and } a^{2}-a c+c^{2}=p \\ 0 & \text { if } \chi_{Q}(p)=-1\end{cases}
$$

the formulas are valid for both $P=P_{k}, \bar{P}_{k}$ and hence for every $P \in \mathcal{P}_{k}$. Consequently, for every positive integer $\mu$ and $P \in \mathcal{P}_{k}$ we have

$$
P \mid \tau(\mu)=\lambda_{k}(\mu) P
$$

where $\lambda_{k}$ is the totally multiplicative function on $\mathbb{N}$ given on prime numbers by (4.18). Thus, we obtain the Euler product expansion of the global orthogonal zeta function of the quadratic form $\mathbf{q}$ with the character $\lambda(\tau(\mu))=\lambda_{k}(\mu)$ :

$$
\begin{aligned}
Z(s, P) & =\sum_{\mu=1}^{\infty} \frac{\lambda_{k}(\mu)}{\mu^{s}} \\
& =\left(1-\frac{\lambda_{k}(3)}{3^{s}}\right)^{-1} \prod_{p \text { primes, } p \neq 3}\left(1-\frac{\lambda_{k}(p)}{p^{s}}+\frac{\chi_{Q}(p) p^{k}}{p^{2 s}}\right)^{-1} .
\end{aligned}
$$

Returning to the ring $\mathcal{O}$ of integral numbers of the field $K=\mathbb{Q}(\rho)$, we can interpret this identity in terms of ideals of $\mathcal{O}$ instead of automorphs of q. For $l$ divisible by 6 , let $\Psi_{l}$ denote the Hecke character of the semigroup of nonzero ideals of $\mathcal{O}$ given by

$$
\Psi_{l}(\mathfrak{a})=\Psi_{l}((\alpha))=\left(\frac{\alpha}{|\alpha|}\right)^{l} .
$$


Then, since

$$
\lambda_{k}(\mathfrak{a})=\lambda_{k}((\alpha))=\alpha^{k}=(\alpha \bar{\alpha})^{k / 2}\left(\frac{\alpha}{|\alpha|}\right)^{k}=N(\mathfrak{a})^{k / 2} \Psi_{k}(\mathfrak{a}) \quad(k \equiv 0(\bmod 6)),
$$

it is not hard to check that the above Euler product can be written in the form

$$
\prod_{\mathfrak{p}}\left(1-\frac{\Psi_{k}(\mathfrak{p})}{N(\mathfrak{p})^{s-k / 2}}\right)^{-1},
$$

where $\mathfrak{p}$ runs through all prime ideals of $\mathcal{O}$. Thus, finally, again we arrive at the identity

$$
Z(s, P)=\zeta_{\mathcal{O}}\left(s-k / 2, \Psi_{k}\right),
$$

where the function

$$
\zeta_{\mathcal{O}}\left(s, \Psi_{k}\right)=\sum_{\mathfrak{a}} \frac{\Psi_{k}(\mathfrak{a})}{N(\mathfrak{a})^{s}}=\prod_{\mathfrak{p}}\left(1-\frac{\Psi_{k}(\mathfrak{p})}{N(\mathfrak{p})^{s}}\right)^{-1},
$$

with $\mathfrak{a}$ running through all nonzero ideals of $\mathcal{O}$, is the Hecke zeta function of the ring $\mathcal{O}$ with the character $\Psi_{k}$. It follows that the Dirichlet series $Z\left(s, \lambda_{k}, \mathbf{q}\right)$ converges absolutely and uniformly in each right half-plane $\operatorname{Re} s \geq 1+k / 2+\epsilon$ with $\epsilon>0$. In every domain of absolute convergence, the function $Z\left(s, \lambda_{k}, \mathbf{q}\right)$ can be presented by means of the Mellin integral of the theta-series $\theta(z ; P, Q)$,

$$
r(2, P, Q) Z(s, P)=\frac{(2 \pi)^{s}}{\Gamma(s)} \int_{0}^{\infty}(\theta(\sqrt{-1} t ; P, Q)-P(0)) t^{s-1} d t
$$

where $r(2, P, Q)$ is the coefficient of $e^{2 \pi i z}$ in the expansion (3.2) of the theta-series, and $\Gamma(s)$ is the gamma function. This representation makes it possible to prove that the zeta function admits meromorphic analytic continuation to the entire $s$-plane and satisfies a functional equation. Alternatively, the analytic properties of the zeta function $\zeta_{\mathcal{O}}\left(s, \Psi_{k}\right)$ can be investigated with the help of Fourier analysis on the adele space of the field $\mathbb{Q}(\sqrt{-3})$.

The general case of positive definite single-class binary quadratic forms can be treated similarly and leads to similar formulas.

\section{REFERENCES}

$[\operatorname{AM}(75)]$ A. N. Andrianov and G. N. Maloletkin, Behavior of theta-series of genus $n$ under modular substitution, Izv. Akad. Nauk SSSR Ser. Mat. 39 (1975), no. 2, 243-258; English transl., Math. USSR-Izv. 9 (1975), 227-241. MR0379382 (52:287)

$[\operatorname{An}(87)]$ A. N. Andrianov, Quadratic forms and Hecke operators, Grundlehren Math. Wiss., vol. 286, Springer-Verlag, Berlin, 1987. MR0884891 (88g:11028)

[An(90)] - Multiplicative properties of solutions of quadratic Diophantine problems, Algebra i Analiz 2 (1990), no. 1, 3-46; English transl., Leningrad Math. J. 2 (1991), no. 1, 1-39. MR.1049904 (91i:11038)

$[\mathrm{An}(91)]-$ Composition of solutions of quadratic Diophantine equations, Uspekhi Mat. Nauk 46 (1991), no. 2, 3-40; English transl., Russian Math. Surveys 46 (1991), no. 2, 1-44. MR1125271 (93e:11049)

$[\operatorname{An}(92)]$ Queen's lectures on arithmetical composition of quadratic forms, Queen's Papers in Pure and Appl. Math., No. 92, Queen's Univ., Kingston, ON, 1992. MR1243633 (95c:11043)

$[\mathrm{An}(93)]+$, Factorizations of integral representations of binary quadratic forms, Algebra i Analiz 5 (1993), no. 1, 81-108; English transl., St. Petersburg Math. J. 5 (1994), no. 1, 71-95. MR.1220490 (94h:11034)

$[\mathrm{An}(94)]+$ Quadratic congruences and rationality of local zeta-series of ternary and quaternary quadratic forms, Algebra i Analiz 6 (1994), no. 2, 1-52; English transl., St. Petersburg Math. J. 6 (1995), no. 2, 199-240. MR1290817(96b:11055) 
[An(95)] , Symmetries of harmonic theta functions of integer-valued quadratic forms, Uspekhi Mat. Nauk 50 (1995), no. 4, 3-44; English transl., Russian Math. Surveys 50 (1995), no. 4, 661-700. MR.1357882(96i:11041)

[An(96)] - Harmonic theta-functions and Hecke operators, Algebra i Analiz 8 (1996), no. 5, 1-31; English transl., St. Petersburg Math. J. 8 (1997), no. 5, 695-720. MR1428987(98a:11053)

[Ca(78)] J. W. S. Cassels, Rational quadratic forms, London Math. Soc. Monogr., vol. 13, Acad. Press, Inc., London-New York, 1978. MR0522835 (80m:10019)

[Fr(91)] E. Freitag, Singular modular forms and theta relations, Lecture Notes in Math., vol. 1487, Springer-Verlag, Berlin, 1991. MR,1165941 (94b:11038)

$[\mathrm{KV}(78)]$ M. Kashiwara and M. Vergne, On the Segal-Shale-Weil representations and harmonic polynomials, Invent. Math. 44 (1978), 1-47. MR0463359 (57:3311)

[Ogg(69)] A. Ogg, Modular forms and Dirichlet series, W. A. Benjamin, Inc., New York-Amsterdam, 1969. MR0256993(41:1648)

St. Petersburg Branch, Steklov Mathematical Institute, Russian Academy of Sciences, Fontanka 27, St. Petersburg 191023, Russia

E-mail address: anandr@pdmi.ras.ru

Received 1/APR/2005

Translated by THE AUTHOR 